\title{
Numerical Studies of Ponderomotive Acceleration and Ion Cyclotron Resonance: Application to Next Generation Electric Thrusters
}

\author{
Fumiko OTSUKA, Tohru HADA, Shunjiro SHINOHARA ${ }^{1)}$, Takao TANIKAWA ${ }^{2)}$ \\ and Takeshi MATSUOKA ${ }^{3, a)}$ \\ Interdisciplinary Graduate School of Engineering Sciences, Kyushu University, Kasuga, Fukuoka 816-8580, Japan \\ ${ }^{1)}$ Institute of Engineering, Tokyo University of Agriculture and Technology, Koganei, Tokyo 184-8588, Japan \\ ${ }^{2)}$ Research Institute of Science and Technology, Tokai University, Hiratsuka, Kanagawa 259-1292, Japan \\ 3) Japan Aerospace Exploration Agency, Sagamihara, Kanagawa 252-5210, Japan
}

(Received 23 March 2012 / Accepted 30 January 2013)

\begin{abstract}
We have examined ponderomotive acceleration/ion cyclotron resonance (PA/ICR) of argon ions by performing test particle simulations. The PA gives rise to the pure parallel acceleration of ions, while the ICR causes the perpendicular ion heating followed by the energy conversion from the perpendicular to the parallel direction in the presence of a divergent background magnetic field. The energy gain by the PA/ICR is classified in terms of the adiabatic parameter, $\Lambda=L_{\mathrm{B}} \Omega_{0} / v_{0 \|}$, where $L_{\mathrm{B}}$ is the axial divergent scale length of the background magnetic field, $\Omega_{0}$ is the ion gyrofrequency at the resonance, and $v_{0 \|}$ is the initial ion drift velocity along the axial magnetic field. For $\Lambda<100$, the energy gain, $\Delta \epsilon$, due to the PA/ICR increases as $\Lambda$ increases. For $\Lambda>100, \Delta \epsilon$ saturates since the increased axial velocity of the ion via the PA reduces the transit time to cross the acceleration region. When the externally applied rf electric field intensity is increased to $1000 \mathrm{~V} / \mathrm{m}$, we find a maximal $60 \%$ increase in the energy gain for the PA/ICR scheme compared with the energy gain by the ICR only. We have applied the $\mathrm{PA} / \mathrm{ICR}$ scheme to the next-generation electric thruster, and have estimated the thrust including ion wall-loss and ion-neutral collisions.
\end{abstract}

(C) 2013 The Japan Society of Plasma Science and Nuclear Fusion Research

Keywords: electrodeless plasma thruster, electromagnetic acceleration, ponderomotive force, ion cyclotron resonance

DOI: $10.1585 /$ pfr.8.1406012

\section{Introduction}

The "electrodeless" configuration for next-generation advanced electric thrusters is very promising because electrodeless electric thrusters utilize no electrodes that erode due to direct contact with the hot plasma propellant, leading to the almost limitless lifetime of the thruster hardware. As a result, research and development of electrodeless electric thrusters have been actively pursued worldwide in various projects, such as the Helicon Electrodeless Advanced Thruster (HEAT) [1-6], the Variable Specific Impulse Magnetoplasma Rocket (VASIMR) [7], and the Double Layer Thrusters (DLT) [8] projects. In all three projects, helicon plasma sources have been adopted as electrodeless radio frequency (rf) plasma sources. Shinohara et al. [2] describe the experimental results of various unique helicon plasma sources with high plasma production efficiencies developed for our HEAT project. While the VASIMR and DLT projects utilize the electrothermal and electrostatic (using plasma double layers) schemes, respectively, for electrodeless plasma accelera-

\footnotetext{
author'se-mail: otsuka@esst.kyushu-u.ac.jp

a) present address: Photon Pioneers Center in Osaka University, Suita, Osaka 565-0871, Japan
}

tion, we have been investigating several electromagnetic plasma acceleration schemes for the HEAT project in order to configure completely electrodeless thrusters with high thruster efficiencies.

In this paper, as part of the HEAT project, we report the numerical results regarding one type of the plasma acceleration schemes, namely the ponderomotive acceleration and ion cyclotron resonance (PA/ICR) scheme. Dodin et al. [9] have proposed a scheme of particle acceleration along a magnetic field due to the ponderomotive potential in an inhomogeneous background magnetic field. We apply their concept to configure electric thrusters. In our work, the effects of ion wall-loss and ion-neutral collisions are included.

The schematic picture of our model is depicted in Fig. 1. The cylindrical chamber (the insulator is assumed in the figure) with the axis parallel to the $z$-direction indicates the plasma acceleration region. A helicon plasma is injected into the chamber from the left-hand side of the chamber. Here, we treat electrons as merely charge neutralizing particles, and only the motion of ions is considered in our test particle simulations as described in Sec. 2.1. In order to impose a linearly polarized transverse 


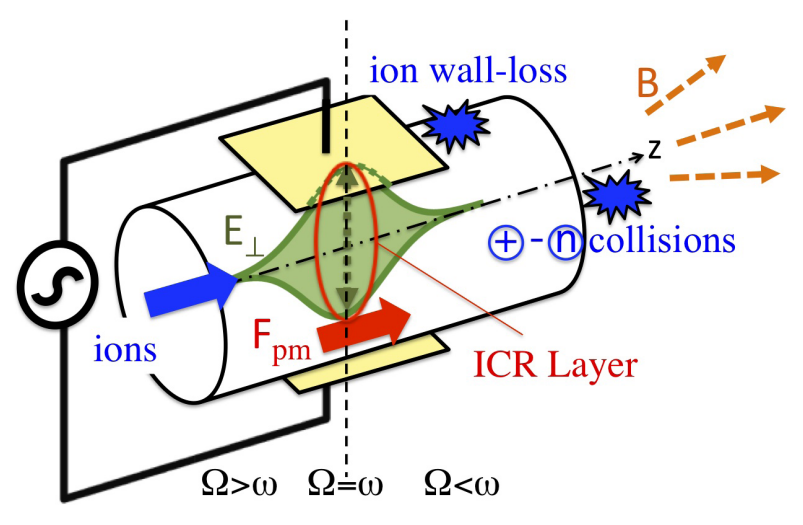

Fig. 1 Schematic picture of the ponderomotive acceleration/ion cyclotron resonance (PA/ICR) scheme.

rf electric field to accelerate ions, a pair of electrodes are placed outside the plasma region (see Fig. 1) so as to avoid the erosion of the electrodes, leading to the electrodeless configuration of the electric thruster. Here, the total penetration of the rf electric field into the plasma is assumed; then, the axial profile of the magnitude of the field, $E(z)$, is given by a Gaussian shape, while the radial profile of the field is assumed to be uniform for simplicity. Then, the ponderomotive potential can be given by [9]

$$
\Phi(z)=\frac{q^{2}}{4 m} \frac{E^{2}(z)}{\omega^{2}-\Omega(z)^{2}},
$$

where $\omega$ is the frequency of the externally applied rf field, and $m, q$, and $\Omega$ are, respectively, the mass, charge and gyrofrequency of the ion species. If the value of $\Omega$ is constant along the $z$-direction (i.e., the background dc magnetic field is uniform in $z$ ), the profile of $\Phi$ is symmetrical with respect to the peak position of $E(z)$. As a result, the directions of the ponderomotive forces with respect to the peak position of $E(z)$ are opposite to each other, resulting in no net acceleration of ions entering into the region of $\Phi$ from the left-hand side of the region. In our scheme, however, the background dc magnetic field is diverging from the left to the right, i.e., the field strength becomes weaker along the $z$-axis; therefore, the value of $\Omega$ decreases along the $z$-axis (see Fig. 2 b). If the resonance point $z_{0}$, at which $\omega=\Omega\left(z_{0}\right)$, coincides with the peak position of $E(z), \Phi$ reverses the sign at $z_{0}$ and the ponderomotive force, $F_{\mathrm{pm}}=-\mathrm{d} \Phi / \mathrm{d} z$, becomes uni-directional. As a result, ions injected from the left-hand side of the chamber, i.e., the stronger magnetic field region, can gain a net energy as they travel through the region of the ponderomotive potential to the right-hand side of the chamber, i.e., the weaker magnetic field region. In short, the parallel (i.e., along the magnetic field) acceleration of ions can be achieved to configure a thruster.

In the above configuration, however, the perpendicular acceleration (or heating) of ions can simultaneously occur due to the ion cyclotron resonance (ICR) effect. The perpendicular energy of the ICR heated ions can then be converted into the parallel energy to contribute to the thrust as the ions travel through the divergent background magnetic field. A well-known application of the ICR to the plasma thruster is the VASIMR. The VASIMR project has been initiated at NASA [7], and the VASIMR type acceleration has also been demonstrated using the HITOP device at Tohoku University in Japan [10, 11]. In the VASIMR type acceleration, large gyroradii of the ICR heated ions tend to cause the strong ion wall-loss at the chamber wall surface that significantly reduces the thrust efficiency, although the VASIMR type experiments $[7,10]$ have succeeded in demonstrating a large acceleration effect of ions within the parameters with which the ion wall-loss can be minimal. Here, we propose the ponderomotive acceleration (PA) scheme as one of the candidates that can avoid the strong ion-wall interaction due to the ICR effect. To summarize, the PA and ICR effects are inseparable, yet the former is preferred since it is less likely to be influenced by ion-wall interactions due to a smaller ion gyroradius compared with the gyroradius of an ICR heated ion.

In this paper, we discuss the ion acceleration due to the PA combined with the ICR mechanism. We have investigated whether or not the PA/ICR acceleration scheme can configure an efficient electric thruster compared with the VASIMR type thruster. Dodin et al. [9] have theoretically analyzed that the ponderomotive effect can be optimized by minimizing the effect of the transverse heating. However, their study has focused on single-particle dynamics. The analysis relevant to the application to electric thrusters under realistic parameters has not been performed. Emsellem [12] has proposed to apply the ponderomotive force to an electric thruster by using a fluid simulation. However, he did not discuss the resultant thrust at all. In this paper, we statistically evaluate the energy gain by the PA/ICR scheme, and classify the results in terms of the adiabatic parameter, $\Lambda=L_{\mathrm{B}} \Omega_{0} / v_{0 \|}$, where $L_{\mathrm{B}}$ is the axial divergent scale length of the background magnetic field, $v_{0 \|}$ is the initial ion drift velocity along the axial magnetic field, and $\Omega_{0}(=\omega)$ is the ion gyrofrequency at $z_{0}$. Furthermore, we include the ion wall-loss and ion-neutral collisions into the model of the PA/ICR scheme, and estimate the thrust as a function of the externally applied rf field intensity.

The organization of this paper is as follows. In Sec. 2, our numerical model is presented. In Sec.3, we show the various numerical results, such as typical orbits, energy evolution, energy gain, and thrust by the PA/ICR. In Secs. 3.1 - 3.4, we discuss the acceleration processes without ion wall-loss and ion-neutral collisions. In Secs. 3.5 and 3.6, we present and discuss the numerical results that include the effects of the ion wall-loss and ion-neutral collisions. In Sec. 4, we summarize the numerical results, and our results are compared with the experimental data of the VASIMR. 


\section{Numerical Model}

We have performed test particle simulations of ions in the localized rf field with the divergent background magnetic field. Ions are assumed to be singly ionized argon. Although the externally applied rf electromagnetic fields we used are similar to those used by Dodin et al. [9], the effects of ion wall-loss and ion-neutral collisions are included in our model so as to make our results more realistic. Considering planned experiments at Tokai University of Japan in mind, we have chosen the parameters such as the chamber size and the background magnetic field intensity. For the ion wall-loss process, the absorbing boundary condition at the wall is used. Our numerical model is explained in detail in this section.

\subsection{Basic equations}

An ion obeys the equation of motion,

$$
m_{\mathrm{Ar}} \frac{\mathrm{d} \boldsymbol{v}}{\mathrm{d} t}=q\left(\boldsymbol{E}_{\mathrm{rf}}+\boldsymbol{v} \times\left(\boldsymbol{B}_{\mathrm{rf}}+\boldsymbol{B}_{0}\right)\right) ; \quad \frac{\mathrm{d} \boldsymbol{r}}{\mathrm{d} t}=\boldsymbol{v},
$$

where $\boldsymbol{v}=\left(v_{x}, v_{y}, v_{z}\right)$ and $\boldsymbol{r}=(x, y, z)$ are, respectively, the velocity and the position vector of the ion, $m_{\mathrm{Ar}}$ is the argon mass, $q=e$ is the charge of the ion, and $e$ is the elementary charge. The rf electric and magnetic fields, $\boldsymbol{E}_{\mathrm{rf}}$ and $\boldsymbol{B}_{\mathrm{rf}}$, are respectively given by

$$
\boldsymbol{E}_{\mathrm{rf}}=E(z) \sin (\omega t) \hat{x} ; \quad \boldsymbol{B}_{\mathrm{rf}}=\frac{E^{\prime}(z)}{\omega} \cos (\omega t) \hat{y},
$$

where the prime denotes $d / d z$ hereafter. The magnitude of $\boldsymbol{E}_{\mathrm{rf}}$ is given by

$$
E(z)=E_{0} \exp \left(-\frac{\left(z-z_{0}\right)^{2}}{L_{\mathrm{E}}^{2}}\right),
$$

where $L_{\mathrm{E}}$ is the width of the Gaussian profile and $E_{0}$ is the maximum intensity at the resonance $z=z_{0}$, where the ion gyrofrequency coincides with the frequency of the externally applied rf fields, $\Omega\left(z_{0}\right)=\omega$. The background magnetic field, $\boldsymbol{B}_{0}$, that must satisfy $\nabla \cdot \boldsymbol{B}_{0}=0$, is divergent along the $z$ axis and can be described by,

$$
\boldsymbol{B}_{0}=\left(-\frac{x}{2} B^{\prime}(z),-\frac{y}{2} B^{\prime}(z), B(z)\right)
$$

where $B(z)$ is given by

$$
B(z)=B_{0}\left(1+\tanh \left(-\frac{z-z_{0}}{L_{\mathrm{B}}}\right)\right) .
$$

Here, $B_{0}$ is the field strength at $\left(0,0, z_{0}\right)$ and $L_{\mathrm{B}}$ is the gradient scale length along $z ; B(z)$ is given by the hyperbolic tangent which goes to zero as $z \rightarrow \infty$.

Figure 2 shows the axial profile of the external fields, (a) $E(z)$ of Eq. (4) and (b) $B(z)$ of Eq. (6), where the parameters used are $z_{0}=0.5 \mathrm{~m}, E_{0}=30 \mathrm{~V} / \mathrm{m}, B_{0}=400 \mathrm{G}$, and $L_{\mathrm{B}}=1 \mathrm{~m}$; then $\Omega\left(z_{0}\right)=\omega \sim 9.66 \times 10^{4} / \mathrm{s}$ corresponding to $15.4 \mathrm{kHz}$.

Three different curves in Fig. 2 (a) correspond to the three different values of $L_{\mathrm{E}}$, namely $L_{\mathrm{E}}=0.1 \mathrm{~m}, 0.4 \mathrm{~m}$ and (a)

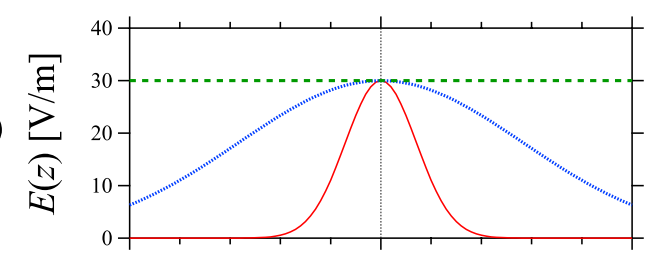

(b)

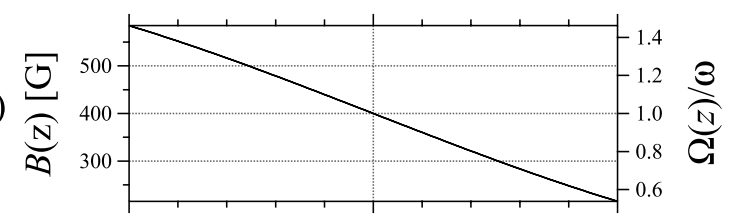

(c)

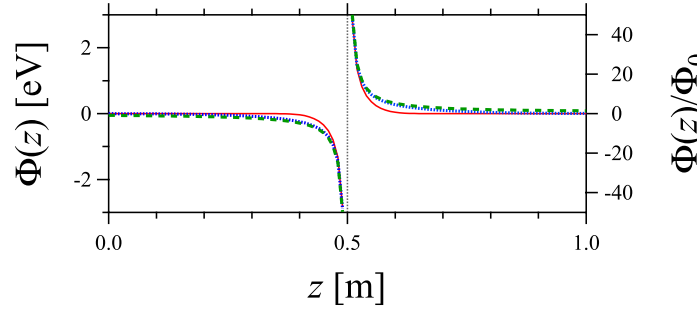

Fig. 2 Spatial profiles of the external fields: (a) the electric field intensity, (b) the axial magnetic field, and (c) the ponderomotive potential. In (a) and (c), the red solid, blue dotted, and green dashed lines represent the cases of $L_{\mathrm{E}}=0.1 \mathrm{~m}, 0.4 \mathrm{~m}$ and $\infty$, respectively. The other parameters used are $E_{0}=30 \mathrm{~V} / \mathrm{m}, L_{\mathrm{B}}=1 \mathrm{~m}, B_{0}=400 \mathrm{G}$, $z_{0}=0.5 \mathrm{~m}$, and $\omega \sim 9.66 \times 10^{4} / \mathrm{s}$ corresponding to $15.4 \mathrm{kHz}$.

$\infty$, respectively. In Fig. 2 (b), the right ordinate shows the ion gyrofrequency normalized by $\omega$. Using $E(z)$ and $B(z)$ above, $\Phi(z)$ in Eq. (1) is plotted in Fig. 2 (c). In the figure, the left ordinate shows the potential in the unit of eV, while the right ordinate shows the potential in a dimensionless form (normalized by using $\Phi_{0}=q^{2} E_{0}^{2} / 4 m_{\mathrm{Ar}} \omega^{2}$ ). Because $\Omega(z)>\omega$ for $z<z_{0}$ and $\Omega(z)<\omega$ for $z>z_{0}$, $\Phi$ reverses the sign at $z=z_{0}$, as can be seen in Fig. 2 (c). As a result, the ponderomotive force along the $z$-direction, $F_{\mathrm{pm}}=-\mathrm{d} \Phi / \mathrm{d} z$, becomes uni-directional so as to always accelerate ions along the positive $z$-direction. Here, we note that $\Phi$ has a singularity at $\Omega\left(z_{0}\right)=\omega \equiv \Omega_{0}$. The infinite $\Phi$ fails to describe the particle motion [9], and the ponderomotive force near gyroresonance has been discussed in detail by Lamb et al. [13].

We integrate Eq.(2) in the given electromagnetic fields described above. The simulation time step we used is $\Delta t=0.01 / \omega$. An important parameter for the PA/ICR mechanism is the adiabatic parameter, $\Lambda=L_{\mathrm{B}} \Omega_{0} / v_{0 \|}$, which is the ratio of $L_{\mathrm{B}}$ to the travel distance of an ion during one gyromotion, $v_{0 \|} / \Omega_{0}$. Large $\Lambda$ means that the particle adiabatically feels the changes of $B(z)$ and $\Phi(z)$. In Sec. 3.3, we discuss the energy gain by the PA/ICR as a function of $\Lambda$. In the calculation in Sec. 3, the parameters, $z_{0}=0.5 \mathrm{~m}, B_{0}=400 \mathrm{G}$, and $L_{\mathrm{B}}=1 \mathrm{~m}$, are fixed, and the values of $L_{\mathrm{E}}$ and $E_{0}$ are varied. 


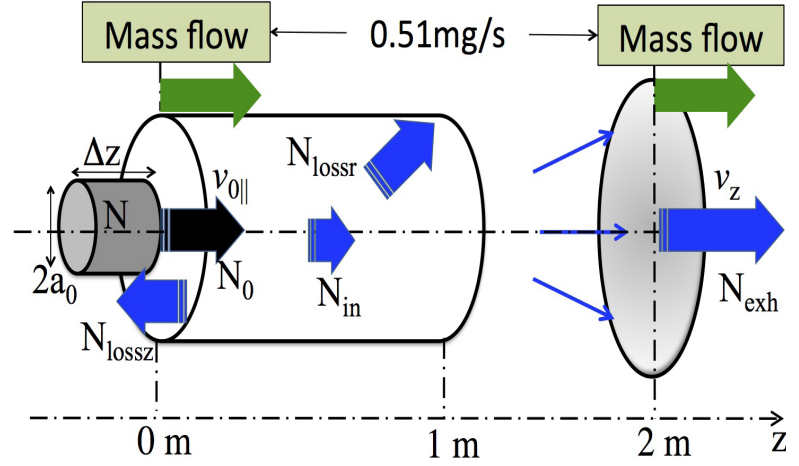

Fig. 3 Schematic picture of the simulation setup.

\subsection{Simulation setup}

Figure 3 shows our simulation setup. Our system consists of mainly two parts: an acceleration region (a cylinder with $0.2 \mathrm{~m}$ diameter and $1 \mathrm{~m}$ length) and an energy conversion region (no bounded space for $1 \mathrm{~m}<z<2 \mathrm{~m}$ ). $N$ test particles of ions are initially located outside the system. Their initial velocities are given by the Maxwellian distribution, shifted by $v_{0 \|}$ along the $v_{z}$ direction, with the temperature $T_{\mathrm{i}}$ that defines the ion thermal velocity of $T_{\mathrm{i}}=m_{\mathrm{Ar}} v_{\mathrm{th}}^{2} / 2$ (Boltzmann's constant is included in the definition of $T_{\mathrm{i}}$ ). The $N$ test particles fill the small cylinder at $z<0 \mathrm{~m}$ indicated by the gray shaded region with the volume $V_{0}=\pi a_{0}^{2} \Delta z$, where $a_{0}$ is the initial plasma radius and $\Delta z\left(=v_{0 \|} \Delta t\right)$ is the axial length of the small cylinder. Each test particle represents $A$ physical particles, and the weight $A(>1)$ is written by $A=V_{0} n_{0} / N$ where $n_{0}$ is the physical number density of the ions. At $t=\Delta t, N_{0}$ particles out of $N$ test particles can cross $z=0 \mathrm{~m}$; then, the mass flow rate can be written by $\dot{m}=m_{\mathrm{Ar}} A N_{0} / \Delta t=\left(N_{0} / N\right)\left(m_{\mathrm{Ar}} n_{0} \pi a_{0}^{2} v_{0 \|}\right)$. We use $T_{\mathrm{i}}=0.067 \mathrm{eV}$ that corresponds to $v_{\text {th }} \sim 570 \mathrm{~m} / \mathrm{s}$ and $v_{0 \|}=400 \mathrm{~m} / \mathrm{s}$; then, $N_{0} / N \sim 0.68$ is numerically obtained by the shifted-Maxwellian. Accordingly, the mass flow rate becomes $\dot{m} \sim 0.51 \mathrm{mg} / \mathrm{s}$, where $a_{0}=0.03 \mathrm{~m}$ and $n_{0}=10^{19} / \mathrm{m}^{3}$ are used. We typically use the number of the test particles, $N=10^{4}$. Hereafter "particle" is used to refer to "test particle".

The injected $N_{0}$ particles are divided into four groups: particles that are absorbed by the side wall, particles that travel to the left-hand side and are absorbed at $z=0 \mathrm{~m}$, particles that remain inside the cylinder, and particles that are exhausted to the right-hand side. The total number of particles in each group is denoted by, $N_{\text {lossr }}, N_{\text {lossz }}, N_{\text {in }}$, and $N_{\text {exh }}$, respectively. In Secs. 3.1 - 3.6 except Sec. 3.5, the trajectories of all of the $N_{0}$ particles introduced into the system at $t=0$ are followed until $N_{\text {in }}$ becomes zero, i.e., all particles introduced into the system are either exhausted out of the system or absorbed by the boundaries. On the other hand, when we investigate the steady-state behavior of particle trajectories in Sec. 3.5, particles are continuously introduced into the system at every time step until $t=1.5 \times 10^{4} \Delta t$; some particles still remain inside the cylinder at the end of the calculation.

\subsection{Statistics of the particle motion}

We explain the statistical estimation of the particle motion. First, we calculate the orbits of individual particles without both the ion wall-loss and ion-neutral collisions in order to understand the statistical behavior of the acceleration process. We take the ensemble average of the particle orbits; then, the average perpendicular and parallel (with respect to the main direction of the background magnetic field) energies, $\epsilon_{\perp}$ and $\epsilon_{\|}$, are evaluated as functions of $z$,

$$
\begin{aligned}
\epsilon_{\perp}(z) & =\frac{m_{\mathrm{Ar}}}{2}\left\langle v_{x}^{2}(\boldsymbol{r}[t])+v_{y}^{2}(\boldsymbol{r}[t])\right\rangle, \\
\epsilon_{\|}(z) & =\frac{m_{\mathrm{Ar}}}{2}\left\langle v_{z}^{2}(\boldsymbol{r}[t])\right\rangle,
\end{aligned}
$$

where \langle\rangle denotes the ensemble average for all particles at the position $z$. Then, the total energy is obtained by $\epsilon(z)=$ $\epsilon_{\perp}(z)+\epsilon_{\|}(z)$. The energy evolution and the energy gain at $z=2 \mathrm{~m}$ are discussed in Secs. 3.2 and 3.3, respectively. We also discuss the phase relation between the externally applied rf field and the single particle gyromotion, since the energy gain of an individual particle depends on this phase relation.

Second, we estimate the thrust including the effects of both the ion wall-loss and ion-neutral collisions in order to obtain more realistic results. The thrust and the mass flow rate of our model electric thruster can be written by [14]

$$
\begin{aligned}
& F=\eta \dot{m} v_{z}, \\
& \dot{m}=m_{\mathrm{Ar}} n \pi a^{2} v_{z}=\frac{N_{0}}{N} m_{\mathrm{Ar}} n_{0} \pi a_{0}^{2} v_{0 \|},
\end{aligned}
$$

where $\eta\left(=N_{\text {exh }} / N_{0}\right)$ is the ratio of the exhausted to the injected particles, $v_{z}$ is the axial exhaust velocity averaged over $N_{\text {exh }}$ particles, $n$ is the number density of ions, and $a$ is the plasma radius. Although the variables, $\eta, v_{z}, n$ and $a$, are the functions of $z$, we only calcuate $\eta$ and $v_{z}$ at $z=2 \mathrm{~m}$ and use the initial mass flow rate, $\dot{m} \sim 0.51 \mathrm{mg} / \mathrm{s}$, since $\dot{m}$ is conserved. In Sec. 3.6, we discuss the behavior of the thrust, $F$, obtained by inserting $\eta$ and $v_{z}$ into Eq. (8a).

\subsection{Theory of perpendicular heating}

The quasi-linear theory (QLT) can describe the perpendicular energy gain due to the ICR in a divergent background magnetic field $[9,15]$. Here, we briefly review the QLT. The theory employs two assumptions: 1) a uniform $\mathrm{rf}$ electric field along the $z$ axis, namely, $E(z)=E_{0}$ in Eq. (4) and 2) no parallel acceleration of ions, namely, $v_{z}=v_{0 \|} \sim$ const. However, it should be noted that, even under the assumption 1), the ponderomotive effect can appear due to the gradient of $\Omega(z)$, leading to an effective parallel acceleration which breaks the second assumption. Within these assumptions, the energy gain can be given by (see Appendix A), 


$$
\begin{aligned}
\Delta \epsilon_{\perp} & =\frac{m_{\mathrm{Ar}}}{2}\left\langle\left|\Delta v_{\perp}^{2}\right|\right\rangle \\
& =\frac{\pi q^{2} E_{0}^{2}}{4 m_{\mathrm{Ar}}} \frac{L_{\mathrm{B}}}{\omega v_{0 \|}}=\pi \Phi_{0} \Lambda,
\end{aligned}
$$

where $\Lambda$ is the adiabatic parameter defined by $\Lambda=$ $L_{\mathrm{B}} \Omega_{0} / v_{0 \|}$. According to Eq. (9), the energy gain is proportional to $\Lambda$. As $\Lambda$ becomes larger, a particle can gyrate many times during the transit time of $L_{\mathrm{B}} / v_{0 \|}$, through the resonance region, leading to a larger energy gain. In Sec. 3.3, we discuss the deviation of the perpendicular energy gain from the QLT due to the PA effect.

\subsection{Ion-neutral collisions}

We consider elastic ion-neutral collisions and charge exchanges with neutral particles. It is assumed that the neutral particles are immobile and uniformly distributed throughout $0<z<2 \mathrm{~m}$. In order to simulate collisions, we employ the Monte Carlo model developed by Vahedi and Surendra [16]. Here, we briefly describe the Monte Carlo model we used. First, a collision takes place if a uniformly distributed random number, $R_{1} \in[0,1]$, is less than a collision probability, $p$. The collision probability is defined as

$$
p=1-\exp \left(-\Delta t n_{\mathrm{n}} I(\epsilon) v_{\mathrm{t}}\right)
$$

where $n_{\mathrm{n}}$ is the number density of neutral particles, $I(\epsilon)$ is the collision cross section which is a function of the particle energy $\epsilon\left(=m_{\mathrm{Ar}} v_{\mathrm{t}}^{2} / 2\right)$, and $v_{\mathrm{t}}$ is the particle speed. The top panel of Fig. 4 shows the cross sections, $I_{\mathrm{el}}$ and $I_{\mathrm{ce}}$, for the elastic collisions and the charge exchanges denoted by the solid and dashed lines, respectively. The cross section, $I_{\mathrm{el}}$, for $\epsilon>0.2 \mathrm{eV}$ is obtained by fitting the experimental data [17] that are indicated in the figure by the empty circles; then, $I_{\mathrm{el}}(\epsilon)$ is given by $I_{\mathrm{el}}(\epsilon)=I_{0}+\alpha \epsilon^{-\beta}$, where $I_{0} \sim$ $1.42 \times 10^{-19} \mathrm{~m}^{2}, \alpha \sim 3.78 \times 10^{-19} \mathrm{~m}^{2}(\mathrm{eV})^{\beta}$, and $\beta \sim 0.28$. Referring to Fig. 5 of [16], we assume $I_{\mathrm{el}}$ for $\epsilon<0.2 \mathrm{eV}$ to be a constant, whose value is $I_{\mathrm{el}} \sim 7.42 \times 10^{-19} \mathrm{~m}^{2}$. Also, we simply define $I_{\mathrm{ce}}=1.3 I_{\mathrm{el}}$, reffering to Fig. 5 of [16]. Then, the probability of the charge exchange, $p_{\mathrm{ce}}$, is larger than that of the elastic collision, $p_{\mathrm{el}}$. We fixed $n_{\mathrm{n}}=10^{19} / \mathrm{m}^{3}$ in the calculation described in Secs. 3.5 and 3.6. The collision frequency, $v_{\text {coll }}=n_{\mathrm{n}} I_{\mathrm{el}}(\epsilon) v_{\mathrm{t}}$, for elastic collisions at $\epsilon=0.2 \mathrm{eV}$ is $v_{\text {coll }} \sim 7.3 \times 10^{3} / \mathrm{s}$; then, $\Omega_{0} / v_{\text {coll }} \sim 13$ that suggests that the gyromotions of ions are dominant compared with the collisions.

Next, the scattering angle, $\theta$, in the center-of-mass frame is determined. Here, we consider two types of scatterings in the center-of-mass frame: isotropic and anisotropic scatterings. In the experiment of $\mathrm{Ar}^{+}-\mathrm{Ar}$ collisions [18], the measured differential cross section has shown highly anisotropic scattering. Vahedi and Surendra [16] have modeled the experimentally obtained anisotropic differential cross section [18]. In our calculation, we use their model differential cross section, $\sigma(\epsilon, \theta)$, written by,
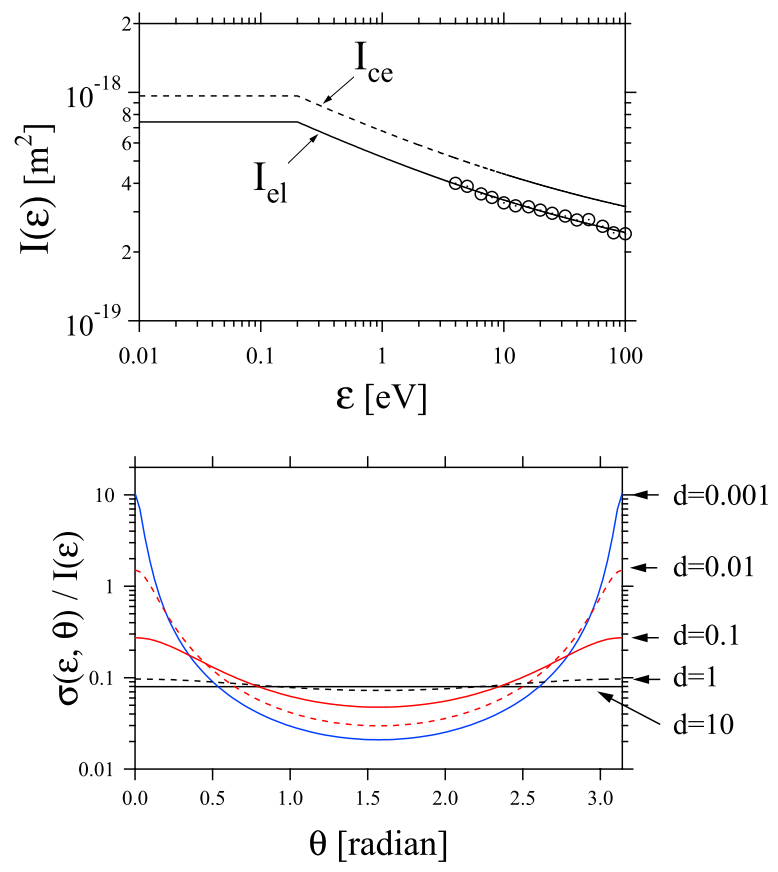

Fig. 4 (Top) Cross section for ion-neutral collisions vs. particle energy. The solid and dashed lines are the cross sections, $I_{\mathrm{el}}$ and $I_{\mathrm{ce}}$, for the elastic collision and the charge exchange, respectively. The empty circles indicate the experimental data [17]. (Bottom) Normalized differential cross section Eq. (11) vs. scattering angle in the centerof-mass frame with different degree of anisotropy represented by a parameter, $d$.

$$
\frac{\sigma(\epsilon, \theta)}{I(\epsilon)}=C \frac{d}{(1+\cos \theta+d)(1-\cos \theta+d)}
$$

where the normalization factor $C$ is given by $C=(1+$ $d) / 2 \pi d \ln (1+2 / d)$, and the parameter $d$ determines the degree of anisotropy: $d \gg 1$ and $d \ll 1$ correspond to the isotropic and anisotropic scatterings, respectively. In the limit of $d \gg 1, \sigma / I$ becomes $1 / 4 \pi$. The model $\sigma(\epsilon, \theta)$ normalized by $I(\epsilon)$ is plotted for several different values of $d$ in the bottom panel of Fig. 4. Vahedi and Surendra [16] concluded that $d$ on the order of $10^{-3}$ could explain the measurements [18] of argon over the relative energy of $2-20 \mathrm{eV}$. Hence, we use $d=0.001$ to calculate the differential cross section, Eq. (11). For the isotropic and anisotropic scatterings, the scattering angles, $\theta$, can be determined by

$$
\begin{aligned}
& \cos \theta=1-2 R_{2} \\
& \cos \theta=(1+d) \frac{1-(d /(d+2))^{1-2 R_{2}}}{1+(d /(d+2))^{1-2 R_{2}}},
\end{aligned}
$$

respectively, using random numbers $R_{2} \in[0,1]$. The second Eq. (12 b) is obtained by using Eq. (11) (see [16] for the details). We assume that the charge exchanges occur when $90^{\circ}<\theta<180^{\circ}$. Accordingly, if $0<R_{1}<p_{\text {el }}$, both the elastic collision and the charge exchange occur; then, $R_{2} \in[0,1]$, i.e. $0^{\circ}<\theta<180^{\circ}$, must be selected. On the 
other hand, if $p_{\mathrm{el}}<R_{1}<p_{\text {ce }}$, only the charge exchange occurs; then, $R_{2} \in[0.5,1]$, i.e., $90^{\circ}<\theta<180^{\circ}$, must be selected. For the charge exchange, the velocities of the ion and the neutral particle are switched after the interaction. For example, when $\theta=160^{\circ}$ is selected in Eq. (12), the scattering angle of the ion becomes $\theta=340^{\circ}$ after the charge exchange interaction. Hence, $\cos \theta \in[0,1]$ is always satisfied after the interaction. The azimuthal scattering angle is uniformly distributed in the interval $[0,2 \pi]$.

\section{Numerical Results}

\subsection{Single particle orbit}

In this subsection, we present the results of numerical simulations without both the ion wall-loss and ion-neutral collisions. Figure 5 shows typical examples of (a) $v_{z}-z$ and (b) $v_{x}-z$ diagrams and (c) trajectories in the $x-z$ plane of ions accelerated by the PA/ICR. In Fig. 5 (c), the background divergent magnetic field lines are also depicted as the dashed lines. The parameters used are $E_{0}=30 \mathrm{~V} / \mathrm{m}$ and $L_{\mathrm{E}}=0.4 \mathrm{~m}$. The particles are initially $(t=0)$ located at $z=0 \mathrm{~m}$ with $v_{x}=v_{y}=0$ and $v_{0 \|}=400 \mathrm{~m} / \mathrm{s}$ (the red solid lines) or $v_{0 \|}=1600 \mathrm{~m} / \mathrm{s}$ (the blue dotted lines). It can be seen in Figs. 5 (a) and 5 (b) that the particles are gradually accelerated by the externally applied rf field both in the parallel and perpendicular directions as they pass through the resonance point, $z_{0}=0.5 \mathrm{~m}$ and make several gyromotions afterward. It is seen in Fig. 5 (c) that the particle's gyroradius increases due to both the increased perpendicular velocity and the decreased background magnetic field intensity as the particle travels toward positive $z$.

Figure 6 shows the evolution of $\epsilon_{\perp}$ and $\epsilon_{\|}$(defined by Eqs. (7a) and (7b) for one particle) for the same runs shown in Fig. 5. The acceleration consists of the following three steps. First, the particle predominantly gains $\epsilon_{\perp}$ via the ICR. Second, the PA makes $\epsilon_{\|}$increase. Finally, $\epsilon_{\perp}$ is converted into $\epsilon_{\|}$(keeping the total energy constant) as the particle travels through the divergent background magnetic field. The increase in both $\epsilon_{\perp}$ and $\epsilon_{\|}$for the $v_{0 \|}=400 \mathrm{~m} / \mathrm{s}$ case is larger than that for the $v_{0 \|}=1600 \mathrm{~m} / \mathrm{s}$ case, since a slower particle can spend a longer time near the resonance point, leading to a larger energy gain by both the ICR and the PA. The ratio of the energy gain by the PA to that by the ICR is around $1 / 3$ in the $v_{0 \|}=400 \mathrm{~m} / \mathrm{s}$ case.

Figure 7 shows the product of $v_{x}$ and $E_{x}$, representing the energy gain of the particle by the ICR. The parameters used are (a) $E_{0}=40 \mathrm{~V} / \mathrm{m}, v_{0 \|}=400 \mathrm{~m} / \mathrm{s}$, and $L_{\mathrm{E}}=0.1 \mathrm{~m}$, (b) $E_{0}=40 \mathrm{~V} / \mathrm{m}, v_{0 \|}=12800 \mathrm{~m} / \mathrm{s}$, and $L_{\mathrm{E}}=0.1 \mathrm{~m}$, and (c) $E_{0}=40 \mathrm{~V} / \mathrm{m}, v_{0 \|}=400 \mathrm{~m} / \mathrm{s}$, and $L_{\mathrm{E}}=\infty$ (axially uniform rf electric field). The corresponding adiabatic parameters are (a) $\Lambda \sim 242$, (b) $\Lambda \sim 7.54$, and (c) $\Lambda \sim 242$; thus, (a) and (c) are adiabatic cases but (b) is a nonadiabatic case. The black solid, blue dotted, and red dashed lines are for the runs with the initial perpendicular velocities of $\left(v_{x}, v_{y}\right)=(400 \mathrm{~m} / \mathrm{s}, 0),(0,400 \mathrm{~m} / \mathrm{s})$, and $(-400 \mathrm{~m} / \mathrm{s}, 0)$, respectively. In Fig. 7 (a), the $v_{x} E_{x}$ is always positive regard- (a)

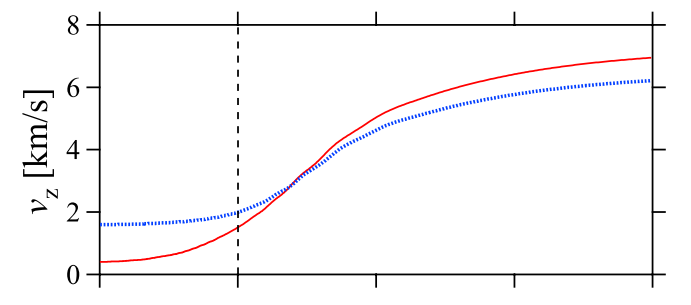

(b)

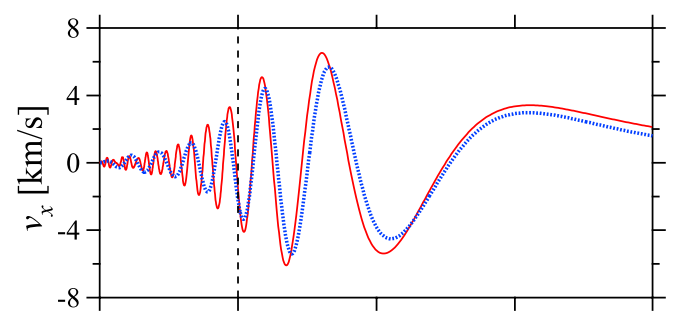

(c)

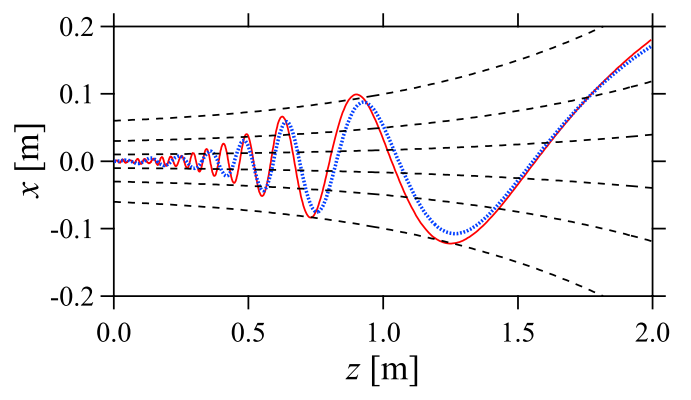

Fig. 5 Typical particle behaviors along the $z$-axis. (a) The axial velocity, $v_{z}$, (b) the perpendicular velocity, $v_{x}$, and (c) the perpendicular position, $x$, of a typical particle with the initial axial velocity $v_{0 \|}=400 \mathrm{~m} / \mathrm{s}$ (the red solid lines) or $v_{0\|\|}=1600 \mathrm{~m} / \mathrm{s}$ (the blue dotted lines) are plotted as functions of $z$. The field parameters used are $E_{0}=30 \mathrm{~V} / \mathrm{m}$ and $L_{\mathrm{E}}=0.4 \mathrm{~m}$. The resonance position, $z_{0}=0.5 \mathrm{~m}$, is indicated by a vertical dotted line in (a) and (b). In (c), the background magnetic field lines are depicted by the dashed curves.

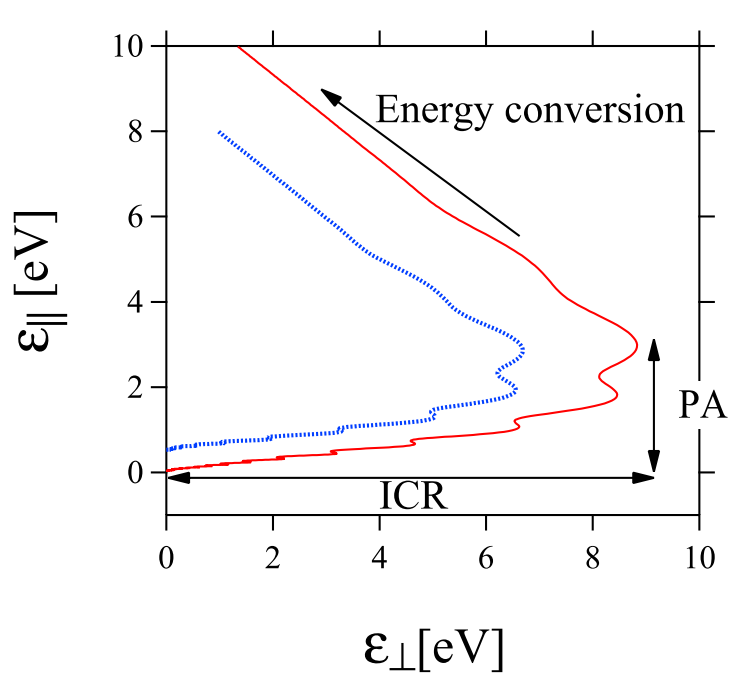

Fig. 6 Phase space trajectories for the parallel and the perpendicular energies, $\epsilon_{\|}$and $\epsilon_{\perp}$, for the same runs as in Fig. 5.

less of the initial particle phase. In Fig. 7 (b), it can be seen that the $v_{x} E_{x}$ oscillates around zero as the particle passes through the region of the rf electric field. The integrals 
(a)

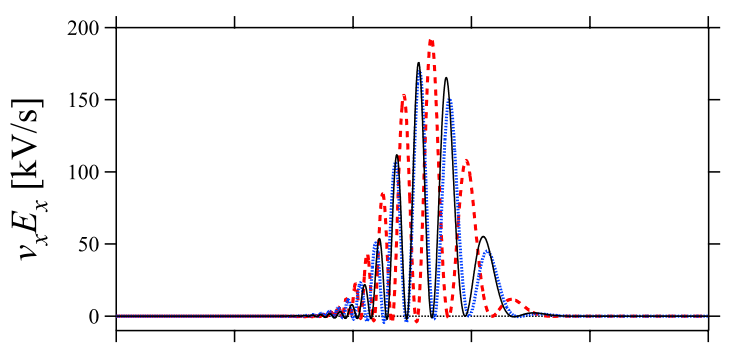

(b)

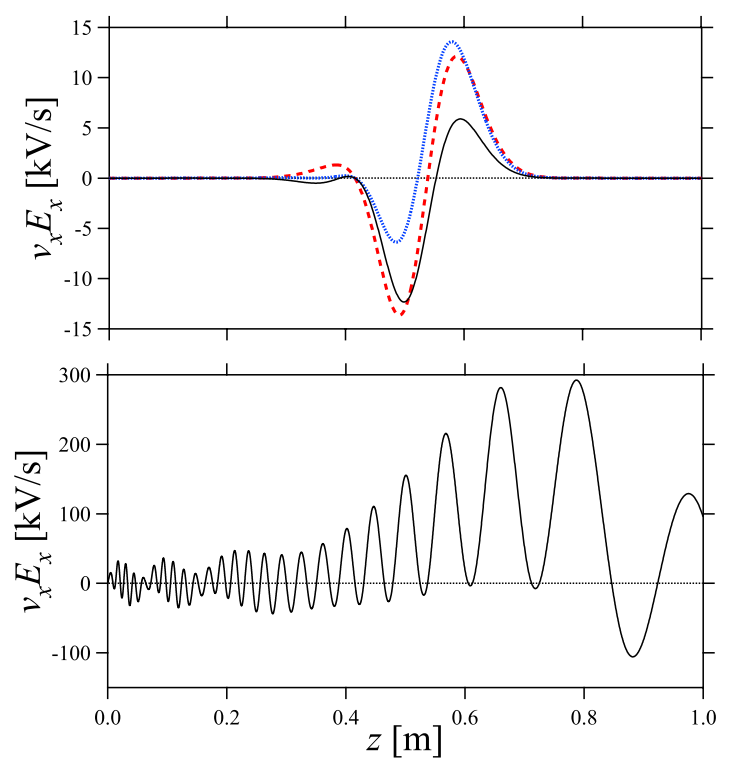

Fig. 7 Product of $v_{x}$ and $E_{x}$ as a function of $z$ for (a) $v_{0 \|}=$ $400 \mathrm{~m} / \mathrm{s}$ and $L_{\mathrm{E}}=0.1 \mathrm{~m}$, (b) $v_{0 \|}=12800 \mathrm{~m} / \mathrm{s}$ and $L_{\mathrm{E}}=0.1 \mathrm{~m}$, and (c) $v_{0 \|}=400 \mathrm{~m} / \mathrm{s}$ and $L_{\mathrm{E}}=\infty$. The other parameter used is $E_{0}=40 \mathrm{~V} / \mathrm{m}$. The black solid, blue dotted, and red dashed lines correspond to the results of the different initial perpendicular velocities, $\left(v_{x}, v_{y}\right)=$ $(400 \mathrm{~m} / \mathrm{s}, 0),(0,400 \mathrm{~m} / \mathrm{s})$, and $(-400 \mathrm{~m} / \mathrm{s}, 0)$ at $t=0$, respectively.

of $v_{x} E_{x}$ in $z$ are approximately zero, suggesting that the overall energy gain is nearly zero as well after the particle passes through the resonance region. Also, the behavior of $v_{x} E_{x}$ does not strongly depend on the initial particle phase. In Fig. 7 (c), for $z<0.4 \mathrm{~m}$, the $v_{x} E_{x}$ oscillates around zero, while for $0.4<z<0.8 \mathrm{~m}$, the $v_{x} E_{x}$ gradually shifts to the positive value, i.e., the particle gains a net energy as it approaches and passes the resonance region. As the particle travels further, the $v_{x} E_{x}$ again oscillates around zero since $\Omega(z)$ deviates from $\omega$ due to the reduced $B(z)$. We notice that many oscillations of $v_{x} E_{x}$ in the adiabatic cases, (a) and (c), contrast with the single oscillation of the nonadiabatic case (b). To summarize, a large $\Lambda$ makes many gyrorotations of the ion which resonates with the oscillations of $E_{x}$, leading to a large perpendicular energy gain due to the ICR.

\subsection{Energy evolution}

In this subsection, we discuss the evolution of the particle energy for the typical trajectories described in the (a)

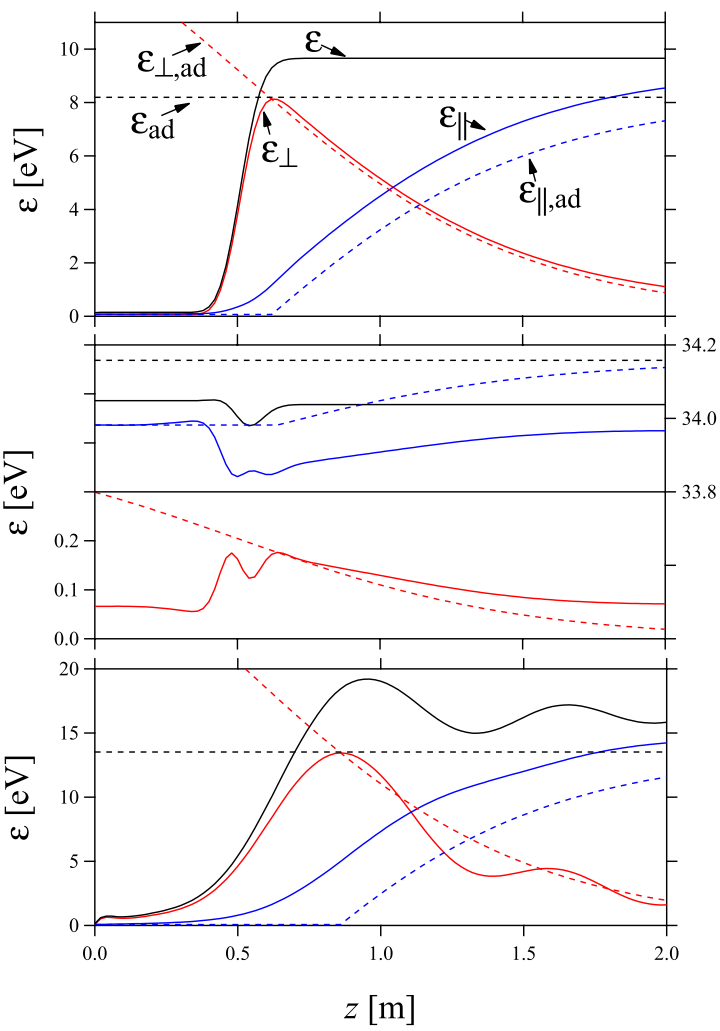

Fig. 8 Evolutions of the ensemble averaged energies, $\epsilon_{\perp}, \epsilon_{\|}$, and $\epsilon$ shown by the red, blue, and black solid lines, respectively. The red, blue and black dashed lines indicate $\epsilon_{\perp, \mathrm{ad}}, \epsilon_{\|, \mathrm{ad}}$ and $\epsilon_{\mathrm{ad}}$ derived using Eq. (14). The parameters used are the same as those used in Fig. 7.

previous subsection. Figure 8 shows the evolution of the particle energies defined by Eq. (7). Parameters used are the same as those used in Fig. 7 except that for the initial ion velocities we assume a shifted-Maxwellian distribution with $T_{\mathrm{i}}=0.067 \mathrm{eV}$, as discussed in Sec. 2.2. The perpendicular $\left(\epsilon_{\perp}\right)$, parallel $\left(\epsilon_{\|}\right)$, and total $(\epsilon)$ energies are shown by the red, blue, and black solid lines, respectively.

First, we examine the case (a) with $\Lambda \sim 242$ and $L_{\mathrm{E}}=0.1 \mathrm{~m}$. Just before the particles reach $z=0.62 \mathrm{~m}$, $\epsilon_{\perp}$ increases sharply due to the ICR. After that, the energy conversion from $\epsilon_{\perp}$ to $\epsilon_{\|}$occurs, keeping $\epsilon$ almost constant. Now we introduce the first adiabatic invariant, $\mu$, and the total energy, $\epsilon$ [9],

$$
\begin{aligned}
\mu & =\frac{\epsilon_{\perp}}{B(z)}, \\
\epsilon & =\mu B(z)+\epsilon_{\|}+\Phi(z),
\end{aligned}
$$

where $B(z)$ is the magnitude of the axial background magnetic field and $\Phi(z)$ is the ponderomotive potential, Eq. (1). If $\mu$ and $\epsilon$ are the invariants of motion,

$$
\begin{aligned}
\mu & \equiv \frac{\epsilon_{\perp}\left(z_{\mathrm{c}}\right)}{B\left(z_{\mathrm{c}}\right)}=\frac{\epsilon_{\perp, \mathrm{ad}}(z)}{B(z)}, \\
\epsilon_{\mathrm{ad}} & \equiv \epsilon_{\perp}\left(z_{c}\right)+\epsilon_{\|, 0}=\epsilon_{\perp, \mathrm{ad}}(z)+\epsilon_{\|, \mathrm{ad}}(z),
\end{aligned}
$$


where $z_{\mathrm{c}}$ is the position at which $\epsilon_{\perp}$ is maximized, $\epsilon_{\|, 0}=$ $m v_{0||}^{2} / 2+T_{\mathrm{i}}$ is the initial parallel energy, and $\Phi(z)=0$ is assumed. The subscript 'ad' represents the energy evolution given by Eq. (14). The constant energy, $\epsilon_{\mathrm{ad}}$, is shown by the black dashed line in Fig. 8. Using the invariants defined above, we obtain the evolution of $\epsilon_{\perp, \text { ad }}$ and $\epsilon_{\|, \text {ad }}$ shown by the red and blue dashed lines in Fig. 8. In Fig. 8 (a), $\epsilon_{\perp}$ and $\epsilon_{\perp, \text { ad }}$ match well, indicating that the energy conversion is in fact adiabatic. On the other hand, $\epsilon$ and $\epsilon_{\|}$are about 1.3 times larger than $\epsilon_{\mathrm{ad}}$ and $\epsilon_{\|, \text {ad }}$, respectively. These discrepancies are due to the effect of the ponderomotive acceleration.

Second, we examine the case (b) with $\Lambda \sim 7.54$ and $L_{\mathrm{E}}=0.1 \mathrm{~m}$. Under these parameters, the typical particles equally experience the loss and gain of energies around $z=0.5 \mathrm{~m}$, as seen in Fig. 7 (b). As a result, the total energy in $z>1 \mathrm{~m}$ slightly decreases from the initial value, representing that the particles are slightly decelerated by the externally applied rf field. Therefore, when $\Lambda$ is small, the particles cannot resonate with the rf field and cannot gain much energy. Also, in the case (b), the evolution of $\epsilon_{\perp}$ cannot be described by the constant $\mu$, since the transit time of $L_{\mathrm{B}} / v_{0 \|}$ is almost equal to the ion gyroperiod of $2 \pi / \Omega_{0}$, i.e. $\Lambda / 2 \pi \sim 1.2$, indicating that the adiabatic condition is not satisfied.

Third, we examine the case (c) with $\Lambda \sim 242$ and $L_{\mathrm{E}}=\infty$. As can be seen in Fig. 7 (c), the typical particles resonate well with the rf field around $0.6 \mathrm{~m}<z<0.8 \mathrm{~m}$; then, $\epsilon$ increases in $0.5 \mathrm{~m}<z<1 \mathrm{~m}$. After that, $\epsilon$ saturates at a constant value with a few oscillations, since $\Omega(z)$ deviates from $\omega$ as the particles travel away from $z_{0}$. In the case (c), $\epsilon_{\perp}$ agrees well with $\epsilon_{\perp, \text { ad }}$, indicating that the energy conversion occurs adiabatically.

\subsection{Energy gain}

We define the energy gain by the ICR and the PA respectively as

$$
\begin{aligned}
\Delta \epsilon_{\perp} & =\epsilon_{\perp}\left(z_{c}\right)-\epsilon_{\perp, 0}, \\
\Delta \epsilon_{\|} & =\Delta \epsilon-\Delta \epsilon_{\perp},
\end{aligned}
$$

where $\Delta \epsilon=\epsilon-\epsilon_{0}$, and the initial perpendicular and initial total energies are respectively $\epsilon_{\perp, 0}=2 T_{\mathrm{i}}, \epsilon_{0}=m v_{0 \|}^{2} / 2+3 T_{\mathrm{i}}$. Figure 9 shows the numerical results of $\Delta \epsilon_{\perp}$ and $\Delta \epsilon_{\|}$. The red circles, blue triangles, and green squares indicate the results of the runs with $L_{\mathrm{E}}=0.1 \mathrm{~m}, 0.4 \mathrm{~m}$, and $\infty$, respectively. The electric field amplitude is $E_{0}=30 \mathrm{~V} / \mathrm{m}$. The solid line in the upper panel represents the QLT in Eq. (9). The energy gains are presented in both the unit of $\mathrm{eV}$ and the dimensionless form normalized by $\Phi_{0}=$ $q^{2} E_{0}^{2} / 4 m_{\mathrm{Ar}} \omega^{2}$. The adiabatic parameter, $\Lambda=L_{\mathrm{B}} \Omega_{0} / v_{0 \|}$, is also shown at the top of each panel.

We have found that both $\Delta \epsilon_{\perp}$ and $\Delta \epsilon_{\|}$decrease as $v_{0 \|}$ increases (or $\Lambda$ decreases) for all runs with different values of $L_{\mathrm{E}}$. These results indicate that both the ICR and the PA occur effectively for large $\Lambda$. The $\Lambda$ dependences of $\Delta \epsilon_{\perp}$
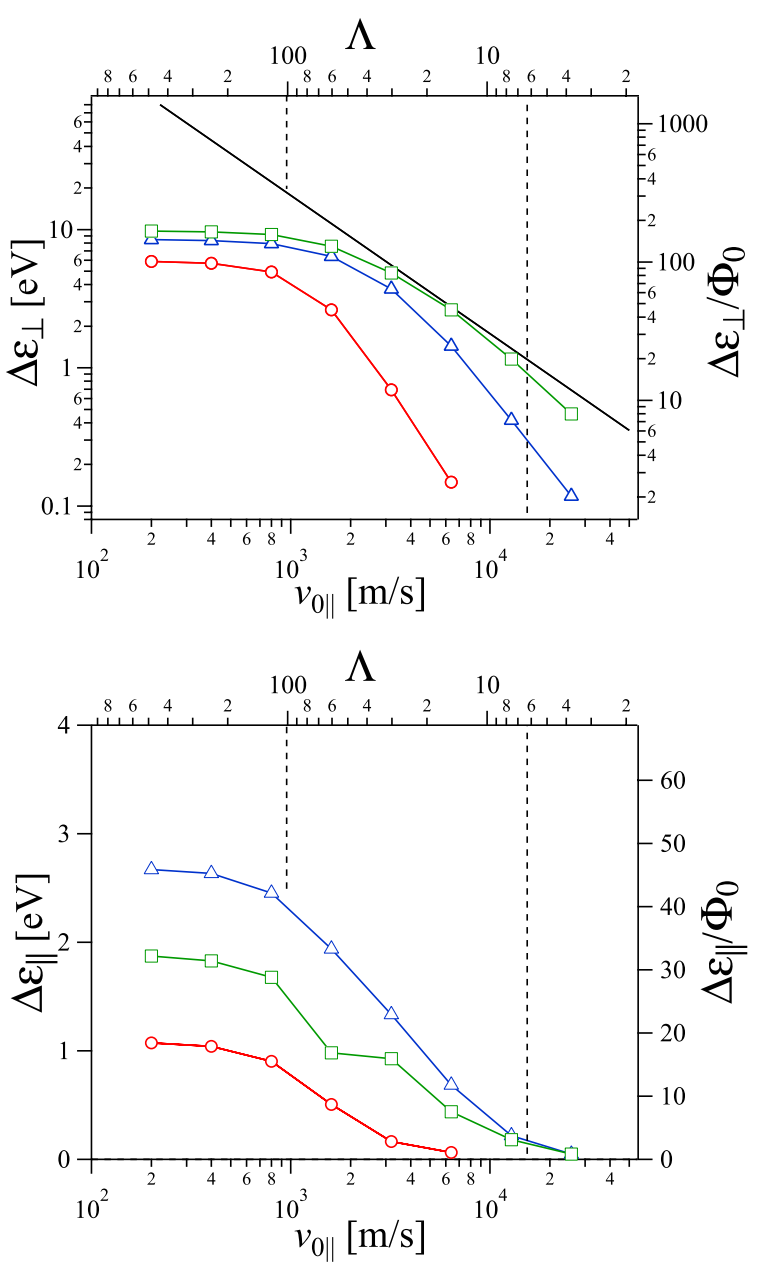

Fig. 9 Energy gain vs. initial drift velocity for the (top) perpendicular and (bottom) parallel components. The red circles, blue triangles, and green squares represent the results for the $L_{\mathrm{E}}=0.1 \mathrm{~m}, 0.4 \mathrm{~m}$, and $\infty$ cases, respectively. The other parameter used is $E_{0}=30 \mathrm{~V} / \mathrm{m}$. The top axis in each panel shows the adiabatic parameter, $\Lambda=L_{\mathrm{B}} \Omega_{0} / v_{0 \|}$. The solid line in the top panel represents the theoretical line of Eq. (9). The vertical dashed lines divide the region of $v_{0 \|}$ into three regimes of the energy gain explained in the text.

and $\Delta \epsilon_{\|}$can be discussed by dividing the scale of $\Lambda$ into three regimes. First, in the middle regime of $100<\Lambda \lesssim 2 \pi$, $\Delta \epsilon_{\perp}$ and $\Delta \epsilon_{\|}$are approximately proportional to $\Lambda$. In this regime, $\Delta \epsilon_{\perp}$ increases with the increase in $L_{\mathrm{E}}$, and the behavior of $\Delta \epsilon_{\perp}$ for $L_{\mathrm{E}}=\infty$ is in good agreement with the QLT. Also, a large $\Lambda$ makes ions keep experiencing a large ponderomotive potential near $z_{0}$, leading to a large energy gain by the PA $\left(\Delta \epsilon_{\|} \propto \Lambda\right)$. Second, in the regime of $\Lambda>100$, both $\Delta \epsilon_{\perp}$ and $\Delta \epsilon_{\|}$saturate. This result indicates that the increased $v_{z}$ via the PA makes the transit time to cross the acceleration region shorter than the time of $L_{\mathrm{B}} / v_{0 \|}$; accordingly, the energy gains by the PA/ICR are suppressed due to the effective parallel acceleration. In this regime, the QLT overestimates $\Delta \epsilon_{\perp}$ since the theory assumes a constant axial velocity $v_{0 \|}$ which is smaller 
than the increased $v_{z}$. Also, in this regime, $\Delta \epsilon_{\|}$is consistent with the ponderomotive potential, $\Phi(z)$. Namely, when $v_{0 \|}=200 \mathrm{~m} / \mathrm{s}(\Lambda \sim 483)$, the saturation level of $\Delta \epsilon_{\|}$varies from 1 to $3 \mathrm{eV}$ which is almost equal to $\Phi(z) \sim 2.8 \mathrm{eV}$ at $\pm 0.01 \mathrm{~m}$ from $z_{0}$ shown (see Fig. 2 (c)). Third, in the regime of $\Lambda \lesssim 2 \pi$, the particles gyrate less than one rotation during the time of $L_{\mathrm{B}} / v_{0 \|}$; then, the PA/ICR is not effective at all. As a result, $\Delta \epsilon_{\|}$approaches to zero, and $\Delta \epsilon_{\perp}$ becomes smaller than the QLT prediction. In particular, when $L_{\mathrm{E}}=0.1 \mathrm{~m}$ and $v_{0 \|}=12800 \mathrm{~m} / \mathrm{s}(\Lambda \sim 7.5)$, we observe the energy loss as can be seen in Fig. 8 (b).

\subsection{Effect of the ponderomotive force}

In order to elucidate the ponderomotive effect quantitatively, we define the index of the ponderomotive effect by

$$
R_{\epsilon}=\frac{\Delta \epsilon}{\Delta \epsilon_{\perp}}=1+\frac{\Delta \epsilon_{\|}}{\Delta \epsilon_{\perp}} .
$$

If $R_{\epsilon}=1$, the energy gain is solely due to the ICR. If $R_{\epsilon}>2$, the energy gain by the PA is larger than that by the ICR. Figure 10 shows (a) the perpendicular and (b) the parallel energy gains, and (c) $R_{\epsilon}$ as functions of $E_{0}$. The red circles and blue triangles represent the results for the $L_{\mathrm{E}}=0.1 \mathrm{~m}$ and $L_{\mathrm{E}}=0.4 \mathrm{~m}$ cases, respectively. The initial drift velocity is $v_{0 \|}=400 \mathrm{~m} / \mathrm{s}$. In Fig. 10 (a), the dashed line shows the QLT result in Eq. (9). We evaluate $R_{\epsilon}$ in Fig. 10 (c) using the values of $\Delta \epsilon_{\perp}$ and $\Delta \epsilon_{\|}$shown in Figs. 10 (a) and 10 (b), respectively.

When $E_{0}$ is increased to $1000 \mathrm{~V} / \mathrm{m}, R_{\epsilon}$ reaches 1.6 for both runs, suggesting a maximum $60 \%$ increase in the energy gain by the PA/ICR scheme compared with that by the ICR only, although the dependences of $R_{\epsilon}$ on $E_{0}$ for the two runs are quite different from each other. It is found that the value of $R_{\epsilon}$ lies between 1 and 2 within the parameter regime we have investigated, suggesting that the energy gain by the ICR is always larger than that by the PA. The maximal value of $R_{\epsilon} \sim 1.6$ is observed in $200 \mathrm{~V} / \mathrm{m} \leq E_{0} \leq 800 \mathrm{~V} / \mathrm{m}$ for $L_{\mathrm{E}}=0.4 \mathrm{~m}$ and at $E_{0}=900 \mathrm{~V} / \mathrm{m}$ for $L_{\mathrm{E}}=0.1 \mathrm{~m}$.

When $E_{0}=2000 \mathrm{~V} / \mathrm{m}$ and $L_{\mathrm{E}}=0.1 \mathrm{~m}$, we observe $R_{\epsilon} \sim 1.8$, suggesting a high efficiency of the PA scheme. However, the particle behavior is highly nonlinear under this condition, so that it is not suitable to use the condition for the electric thruster application as explained below. Let us look at Fig. 11 that shows the $v_{z}-z$ phase space trajectories of five particles which are randomly selected from the initially shifted-Maxwellian distributed particles. Figures 11 (a) and 11 (b) show the results for $L_{\mathrm{E}}=0.1 \mathrm{~m}$ and $L_{\mathrm{E}}=0.4 \mathrm{~m}$, respectively. The other parameters used are $E_{0}=1000 \mathrm{~V} / \mathrm{m}$ and $v_{0 \|}=400 \mathrm{~m} / \mathrm{s}$. For the case of $L_{\mathrm{E}}=0.1 \mathrm{~m}$ (Fig. $\left.11(\mathrm{a})\right)$, the particles injected from $z=0 \mathrm{~m}$ are reflected twice by the ponderomotive potential around $z_{0}=0.5 \mathrm{~m}$; then they pass through the region of the potential toward positive $z$. Especially, the two of the five particles reflected at $z \sim 0.2 \mathrm{~m}$ can gain a larger parallel (a)

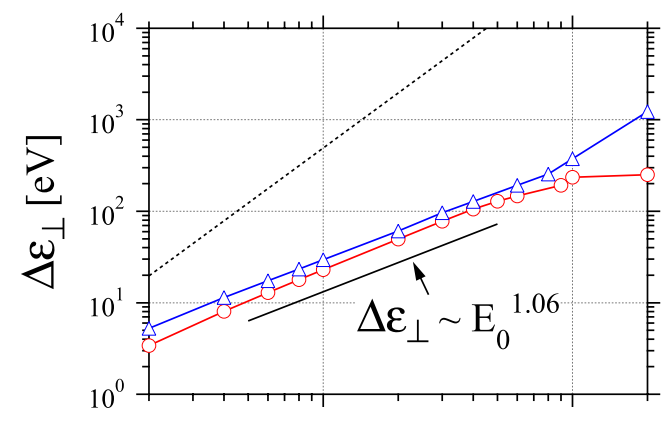

(b)

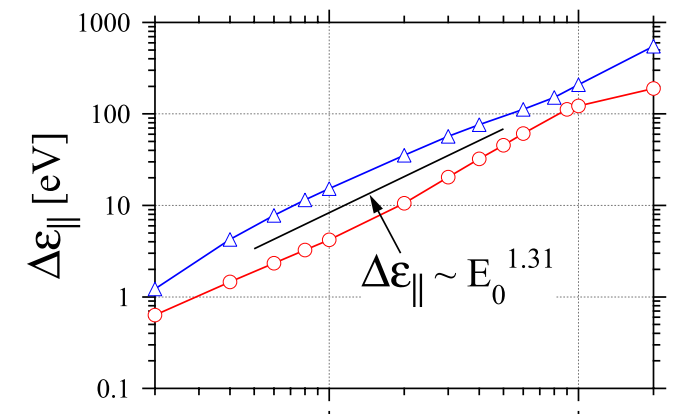

(c)

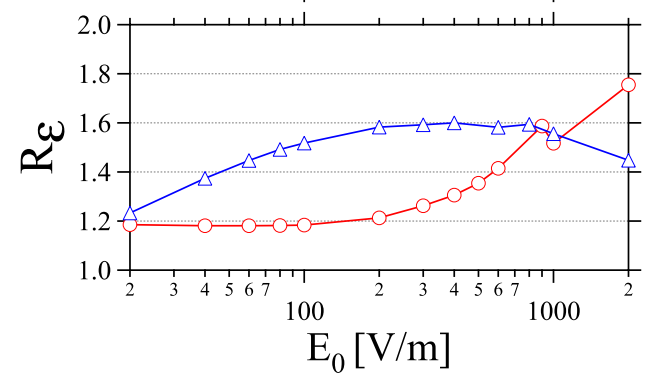

Fig. 10 (a) Perpendicular and (b) parallel energy gains, and (c) the index of the ponderomotive effect $R_{\epsilon}$ defined by Eq. (16) as functions of the electric field intensity, $E_{0}$. The red circles and blue triangles represent the results for the $L_{\mathrm{E}}=0.1 \mathrm{~m}$ and $0.4 \mathrm{~m}$ cases, respectively. The initial drift velocity of $v_{0 \| \mid}=400 \mathrm{~m} / \mathrm{s}$ is used.

(a)

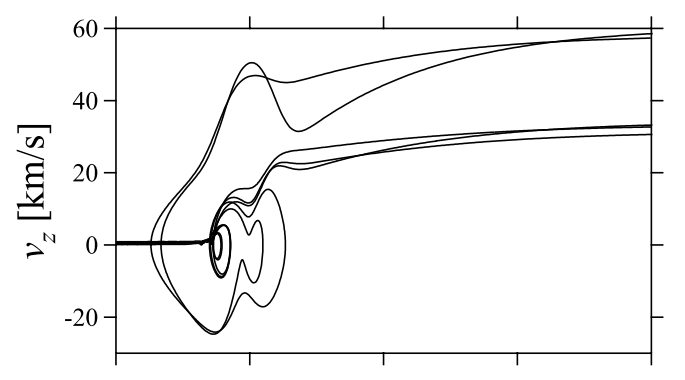

(b)

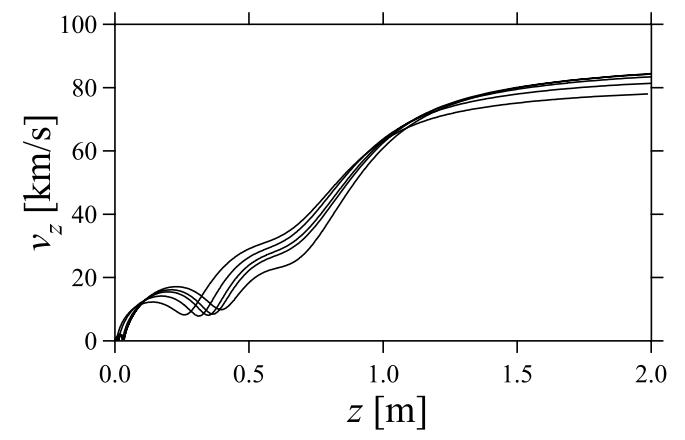

Fig. 11 Typical five trajectories in the $v_{z}-z$ phase space for (a) $L_{\mathrm{E}}=0.1 \mathrm{~m}$ and (b) $L_{\mathrm{E}}=0.4 \mathrm{~m}$. The other parameters used are $E_{0}=1000 \mathrm{~V} / \mathrm{m}$ and $v_{0 \|}=400 \mathrm{~m} / \mathrm{s}$. 
energy than the rest of the five particles; thus, the large $R_{\epsilon}$ can be obtained for $L_{\mathrm{E}}=0.1 \mathrm{~m}$ and $E_{0}=1000 \mathrm{~V} / \mathrm{m}$. This behavior of reflected particles is complex, and cannot be described by a simple uni-directional ponderomotive force based on Eq. (1). In contrast to this behavior, for the case of $L_{\mathrm{E}}=0.4 \mathrm{~m}$ (Fig. 11 (b)), the particles never reflect back. The second reflection in Fig. 11 (a) does not always occur for the shifted-Maxwellian particles. A small fraction of the particles $(\sim 1 \%)$ cannot return to the potential region; they are simply lost at $z=0 \mathrm{~m}$. When $E_{0}$ is further increased up to $2000 \mathrm{~V} / \mathrm{m}$ for $L_{\mathrm{E}}=0.1 \mathrm{~m}, 53 \%$ of the shifted-Maxwellian particles are lost at $z=0 \mathrm{~m}$ due to the reflection by the large potential; as a result, the thrust is significantly reduced. Therefore, there is a limit to utilize the ponderomotive potential as a source of thrust in the strong electric field regime, $E_{0}>1000 \mathrm{~V} / \mathrm{m}$. The detailed investigations regarding this complex behavior due to a large ponderomotive potential are left for future work. The $E_{0}$ dependences of $\Delta \epsilon_{\perp}$ and $\Delta \epsilon_{\|}$are found to be $\Delta \epsilon_{\perp} \propto E_{0}^{1.06}$ and $\Delta \epsilon_{\|} \propto E_{0}^{1.31}$ for $20<E_{0}<1000 \mathrm{~V} / \mathrm{m}$, respectively, by taking the average of two sets of runs. The former relation is quite different from the QLT result of $\Delta \epsilon_{\perp} \propto E_{0}^{2}$; thus, the energy gain should be discussed beyond the limit of the QLT.

\subsection{Effect of ion-neutral collisions}

Now we include the ion wall-loss and ion-neutral collisions in our numerical simulations. At every time step, particles are introduced into the system from the left-hand side boundary. Figure 12 shows the snap shots of particles in the $v_{z}-z$ and $x-z$ spaces at $t=15000 \Delta t$. The parameters used are the same as those used in Figs. 7 (a) and $8(\mathrm{a})$. In the top panel, the averaged exhaust velocity, $v_{z}$, at $z=2 \mathrm{~m}$ is approximately $2 \mathrm{~km} / \mathrm{s}$. While in Fig. 8 (a), the averaged axial energy is $8 \mathrm{eV}$, corresponding to $v_{z} \sim 6.2 \mathrm{~km} / \mathrm{s}$. Hence, the effect of ion-neutral collisions is to reduce the exhaust velocity $v_{z}$. It can also be seen that ion-neutral collisions can cause the particles to have negative $v_{z}$ around $z_{0}=0.5 \mathrm{~m}$. Then, some of these particles with negative $v_{z}$ are reflected by the potential and travel toward the right-hand side, while the rest of these particles continue to travel toward the left-hand side and are eventually lost at the wall $(z=0 \mathrm{~m})$.

Figure 13 is the same as Fig. 12, except that the anisotropic scattering is assumed with the degree of anisotropy $d=0.001$ in Eq. (11). The averaged $v_{z}$ at $z=2 \mathrm{~m}$ is about $4 \mathrm{~km} / \mathrm{s}$ which is larger than the value of $v_{z}$ for the isotropic scattering case. Also, the number of particles with negative $v_{z}$ around $z_{0}=0.5 \mathrm{~m}$ is less than that for the isotropic scattering case. Furthermore, from the bottom panel of Fig. 13, it can be seen that the ions gyrate around $z=1.0 \mathrm{~m}$, whereas, for the isotropic scattering case, the feature of gyromotion is completely washed out by the ion-neutral collisions in the region of $z>1.0 \mathrm{~m}$ (see the bottom panel of Fig. 12). Therefore, the isotropic
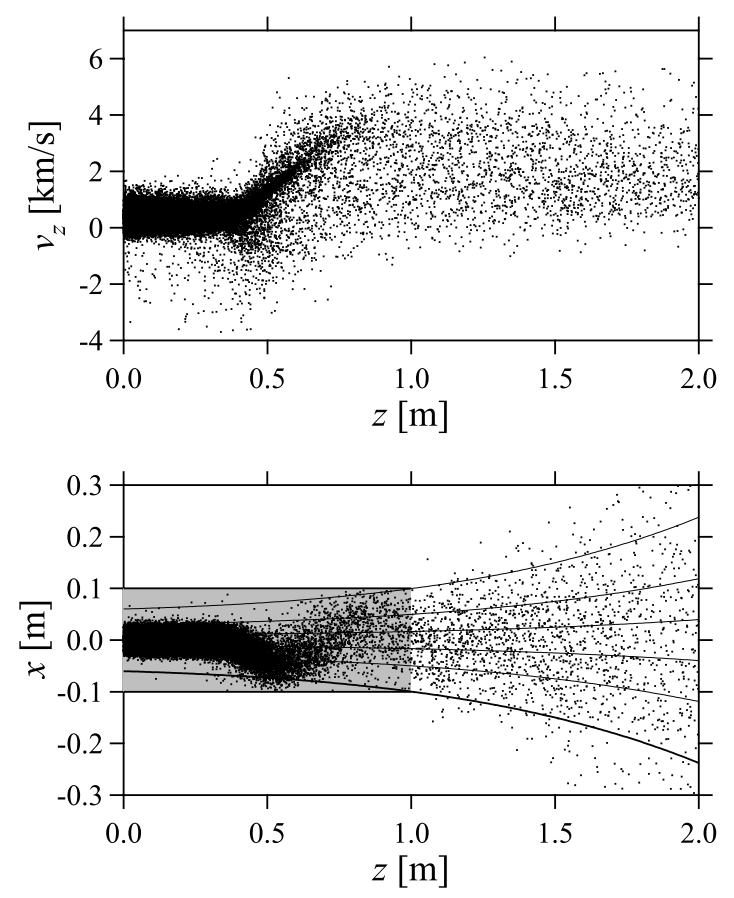

Fig. 12 Snap shots of continuously injected particles at $t=$ $15000 \Delta t$ in (top) $v_{z}-z$ and (bottom) $x-z$ spaces, including the ion wall-loss and ion-neutral collisions with the isotropic scattering. The parameters used are $E_{0}=$ $40 \mathrm{~V} / \mathrm{m}, L_{\mathrm{E}}=0.1 \mathrm{~m}, v_{0 \|}=400 \mathrm{~m} / \mathrm{s}$, and $T_{\mathrm{i}}=0.067 \mathrm{eV}$. In the bottom panel, the shaded region and the solid lines indicate the inside area of the chamber and the background magnetic field lines, respectively.
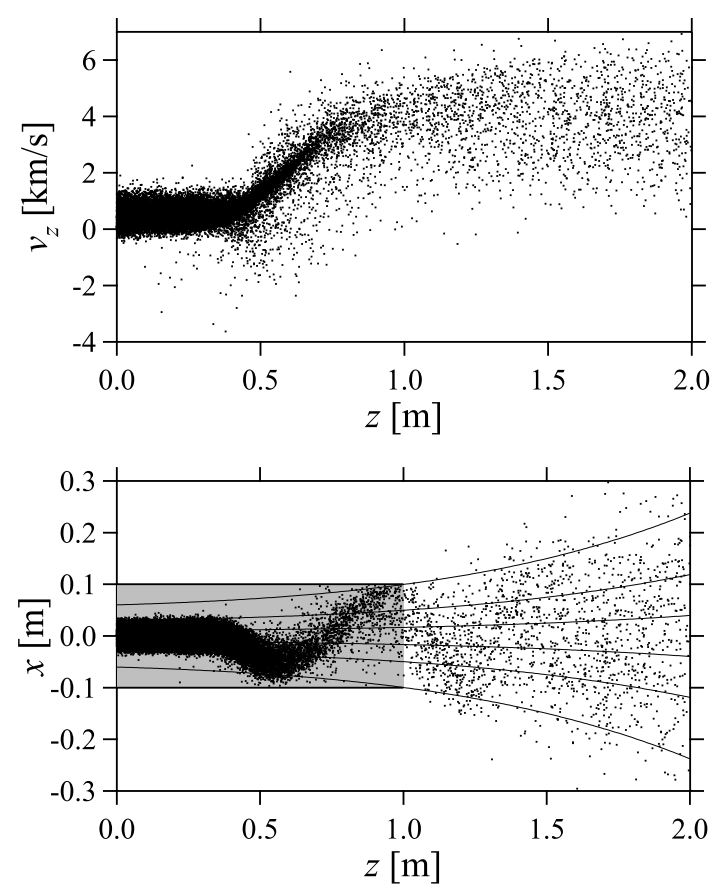

Fig. 13 The same as Fig. 12 but for the anisotropic scattering. 
scattering has greater influence on the particle motion than the anisotropic scattering does. The effect of ion-neutral collisions on thrust is discussed in the next subsection.

\subsection{Thrust estimation}

We estimate the thrust in Eq. (8) by using the exhaust velocity, $v_{z}$, and the exhaust rate, $\eta$, at $z=2 \mathrm{~m}$. We follow the trajectories of the particles that are initially introduced into the system from the left hand-side boundary. Figure 14 shows the numerical results of $v_{z}, \eta$, and the thrust $F$ as functions of $E_{0}$. The parameters used are $L_{\mathrm{E}}=0.1 \mathrm{~m}$, $v_{0 \|}=400 \mathrm{~m} / \mathrm{s}$, and $T_{\mathrm{i}} \sim 0.067 \mathrm{eV}$, and the ion and neutral particle densities are $n_{0}=n_{\mathrm{n}}=10^{19} / \mathrm{m}^{3}$. The results of different runs with and without the ion wall-loss and the ion-neutral collisions are shown by using the different symbols. The solid lines in Figs. 14 (a) and 14 (c) represent the theoretical results based on the QLT as discussed below. In the theory, we assume that the perpendicular energy is completely converted into the axial energy, i.e., $m v_{z}^{2} / 2=\Delta \epsilon_{\perp}$, where Eq. (9) is used to evaluate $\Delta \epsilon_{\perp}$ and the initial drift velocity is neglected due to $v_{z} \gg v_{0\|\|}$. We also assume that $\eta=1$. Accordingly, $v_{z}$ and $F$ in Eq. (8) for the QLT are written by

$$
\begin{aligned}
& v_{z}=\sqrt{\frac{\pi \Lambda}{2}} \frac{E_{0}}{B_{0}}, \\
& F=\left(\frac{N_{0}}{N} m_{\mathrm{Ar}} n_{0} \pi a_{0}^{2} v_{0 \|}\right) v_{z} .
\end{aligned}
$$

The part within the parenthesis in Eq. (17b) represents the mass flow rate described in Sec. 2.2.

First, we examine the exhaust velocity, $v_{z}$, shown in Fig. 14 (a). All numerically obtained $v_{z}$ for the different conditions are increasing functions of $E_{0}$ with a similar increase rate. We find $v_{z} \propto E_{0}^{0.58}$ by taking the average of three sets of runs excluding the run with the ion wallloss and without ion-neutral collisions (shown by the green squares). When $E_{0}=400 \mathrm{~V} / \mathrm{m}$, the ratios of $v_{z}$ with the isotropic and the anisotropic ion-neutral collisions (shown by the red triangles and the blue inverted triangles, respectively) to $v_{z}$ without ion-neutral collisions (shown by the black circles) are 0.35 and 0.59 , respectively, showing that the isotropic and anisotropic ion-neutral collisions reduce $v_{z}$ down to $35 \%$ and $59 \%$, respectively, with respect to the $v_{z}$ without collisions.

Next, we discuss the behaviors of $\eta$ shown in Fig. 14 (b). If the ion wall-loss is included in the simulation without taking into account the ion-neutral collision effect (shown by the green squares), $\eta=0$ for $E_{0}>100 \mathrm{~V} / \mathrm{m}$. This means that all particles are lost at the wall of the chamber because the ICR effect with a large $E_{0}$ is so effective that the gyrating ions with increased gyroradii can eventually intersect with the chamber wall. More detailed discussion of the ion wall-loss will be given in the next section. When the ion-neutral collisions are included, $\eta$ becomes finite even for $E_{0}>100 \mathrm{~V} / \mathrm{m}$ since the collisions reduce the perpendicular velocity. (a)

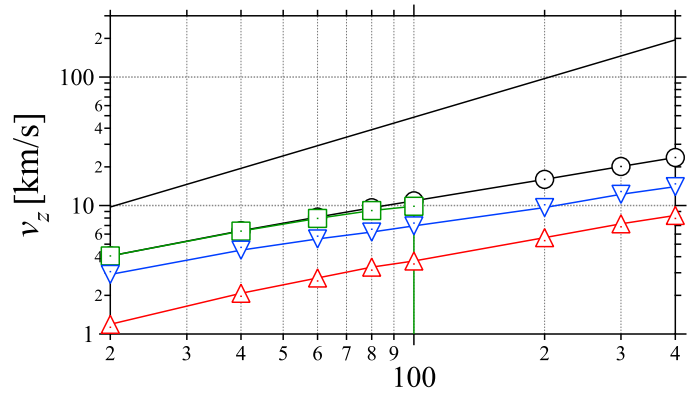

(b)

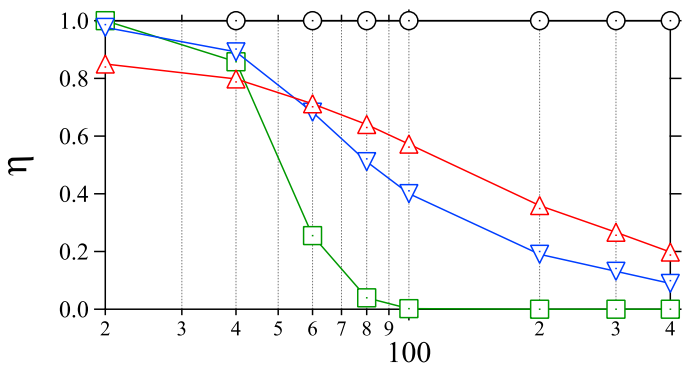

(c)

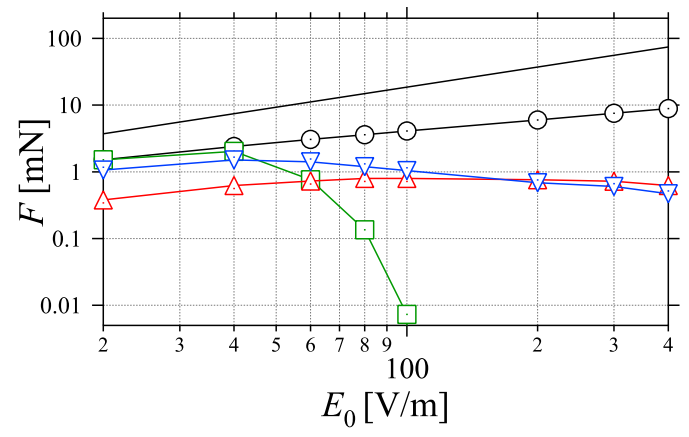

Fig. 14 (a) Axial exhaust velocity $v_{z}$, (b) exhaust rate $\eta$, and (c) thrust $F$ as functions of electric field intensity $E_{0}$. The symbols represent the numerical results without the wall-loss and without the collisions (o), with the wallloss and without the collisions ( $\square$ ), with the wall-loss and with the isotropic collisions $(\Delta)$, and with the wallloss and with the anisotropic collisions $(\nabla)$. The solid lines show the QLT based exhaust velocity and thrust in Eq. (17).

Finally, we examine $E_{0}$ dependences of $F$ for various cases shown in Fig. 14 (c). When the ion wall-loss and ionneutral collisions are both absent, $F$ increases with the increase in $E_{0}$. For this case, $\eta=1$ and $F$ is determined by $v_{z}$ only. When the ion wall-loss and ion-neutral collisions are both included, $v_{z}$ is an increasing function of $E_{0}$, whereas $\eta$ is a decreasing function of $E_{0}$. The consequence is that, in the region of $E_{0}>40 \mathrm{~V} / \mathrm{m}, F$ is almost constant for the isotropic scattering case while it is a weakly decreasing function of $E_{0}$ for the anisotropic scattering case. From the experiment [18], the anisotropic scattering is more realistic than the isotropic scattering. We find the maximal thrust for the anisotropic scattering case to be $F=1.5 \mathrm{mN}$ for $E_{0}=40 \mathrm{~V} / \mathrm{m}$.

\section{Summary and Discussion}

We have examined the PA/ICR of argon ions by per- 
Table 1 Comparison of typical parameters among three different systems. The values of the gyroradius in the 7 th row are estimated by using the gyrofrequencies and the numerically or experimentally obtained velocities in the 5th and 6th rows, respectively.

\begin{tabular}{l||c|c|c}
\hline & present simulation & HITOP [10,11] & VASIMR [7] \\
\hline \hline device raidus & $0.1 \mathrm{~m}$ & $0.4 \mathrm{~m}$ & $0.178 \mathrm{~m}$ \\
\hline magnetic field intensity & $0.04 \mathrm{~T}$ & $0.05 \mathrm{~T}$ & $0.26 \mathrm{~T}$ \\
\hline ion species & $\mathrm{Ar}$ & $\mathrm{He}$ & Deuterium \\
\hline gyrofrequency, $\Omega_{0}$ & $10^{5} / \mathrm{s}(15 \mathrm{kHz})$ & $1.2 \times 10^{6} / \mathrm{s}(200 \mathrm{kHz})$ & $1.2 \times 10^{7} / \mathrm{s}(2 \mathrm{MHz})$ \\
\hline achieved velocity & $v_{z} \sim 10 \mathrm{~km} / \mathrm{s}$ & $v_{\perp} \sim 70 \mathrm{~km} / \mathrm{s}(100 \mathrm{eV})$ & $v_{z} \sim 150 \mathrm{~km} / \mathrm{s}$ \\
\hline gyroradius, $\rho \sim v_{\perp} / \Omega_{0} \sim v_{z} / \Omega_{0}$ & $0.1 \mathrm{~m}$ & $0.058 \mathrm{~m}$ & $0.012 \mathrm{~m}$ \\
\hline
\end{tabular}

forming test particle simulations. When a localized rf field is applied to a plasma in the background divergent magnetic field, both the ICR and the PA simultaneously occur near the resonance point $\left(\omega=\Omega\left(z_{0}\right) \equiv \Omega_{0}\right)$. The ICR causes the perpendicular heating, while the PA causes the parallel acceleration of the ions. The energy conversion from the perpendicular to the parallel direction further enhances the parallel energy as the ions travel through the divergent magnetic field.

The VASIMR utilizes the ICR acceleration to configure an electric thruster. In the VASIMR type acceleration, the PA should also occur since the ICR and the PA are inseparable. In the VASIMR, electromagnetic ion cyclotron (EMIC) waves are applied to heat the ions [7]. We should verify whether or not the PA also operates in this configuration. In this paper, we have examined how the PA can contribute to the energy gain of the ions in our PA/ICR scheme in which the non-propagating rf field is applied.

The energy gain of ions, $\Delta \epsilon$, can be divided into two components: $\Delta \epsilon_{\perp}$ due to the ICR and $\Delta \epsilon_{\|}$due to the PA. Then, we have classified the behaviors of $\Delta \epsilon_{\perp}$ and $\Delta \epsilon_{\|}$into three distinct regimes by using the adiabatic parameter, $\Lambda=L_{\mathrm{B}} \Omega_{0} / v_{0 \|}$ (Fig. 9). For $\Lambda<100$, both $\Delta \epsilon_{\perp}$ and $\Delta \epsilon_{\|}$ increase as $\Lambda$ increases since the ions can make many gyrorotations to acquire the energy near the resonance point. For $\Lambda>100$, both $\Delta \epsilon_{\perp}$ and $\Delta \epsilon_{\|}$saturate since the increased axial velocity via the PA reduces the transit time to cross the acceleration region, so that the acceleration effect is offset. In this regime, the nonlinear interaction between the ICR and the PA occurs; consequently, the quasi-linear theory (QLT) does not correctly describe the behavior of $\Delta \epsilon_{\perp}$.

When the externally applied rf field intensity, $E_{0}$, is increased to $1000 \mathrm{~V} / \mathrm{m}$, we have found $\Delta \epsilon / \Delta \epsilon_{\perp}=1.6$, indicating that the PA raises the energy gain in the PA/ICR scheme up to $60 \%$ compared with the ICR effect only. Therefore, the PA plays an essential role in the ion acceleration by our PA/ICR scheme, although $\Delta \epsilon_{\perp}$ is always larger than $\Delta \epsilon_{\|}$(Fig. $10(\mathrm{c})$ ). These results are useful when the PA/ICR scheme is applied to the electric thruster.

We have examined the thrust by the PA/ICR scheme including the effects of the ion wall-loss and ion-neutral collisions (Fig. 14). For ion-neutral collisions, the scattering in the center-of-mass frame is assumed to be anisotropic rather than isotropic since the anisotropic scattering can better describe the experimental results [18]. The ion-neutral collision effect reduces the exhaust velocity, $v_{z}$, down to $59 \%$ of the value obtained without including the ion-neutral collision effect. The maximum thrust of $F=1.5 \mathrm{mN}$ is obtained at $E_{0}=40 \mathrm{~V} / \mathrm{m}$. When $E_{0}>40 \mathrm{~V} / \mathrm{m}$, the thrust is a weakly decreasing function of $E_{0}$ because of the balance between the increased $v_{z}$ and the decreased exhaust rate due to the wall-loss with the increase in $E_{0}$.

Now, we discuss the ion wall-loss process in detail. Table 1 shows the comparison between our simulation and two VASIMR type experiments. First, we roughly estimate the gyroradius, $\rho$, in our simulation. If $v_{z}$ is equal to the perpendicular velocity increased by the ICR, the gyroradius can be written as $\rho \sim v_{z} / \Omega_{0}$. In our simulation, by using $\Omega_{0} \sim 10^{5} / \mathrm{s}$ and $v_{z}=10 \mathrm{~km} / \mathrm{s}$ at $E_{0}=100 \mathrm{~V} / \mathrm{m}$ (under this condition, all particles are lost at the radial wall if the effect of ion-neutral collisions is not taken into account), we obtain $\rho \sim 0.1 \mathrm{~m}$ which is equal to the chamber radius. Therefore, when the gyroradius of the ICR heated ion is comparable to the chamber radius, the thrust will be significantly reduced due to the strong ion-wall loss.

Next, we estimate $\rho$ in the two VASIMR type experiments. The HITOP experiment has achieved the perpendicular thermal velocity of $\sim 70 \mathrm{~km} / \mathrm{s}$ that corresponds to the perpendicular energy of $100 \mathrm{eV}$ for Helium propellant, while the VASIMR experiment has achieved the exhaust velocity of $150 \mathrm{~km} / \mathrm{s}$ for Deuterium propellant. In both experiments, we found that $\rho$ is sufficiently less than the chamber radius, so that the ion wall-loss can be avoided. We note that the value of $\Omega_{0}$ in two experiments are larger than $\Omega_{0}$ in our simulation because they used He and $\mathrm{D}_{2}$ as propellant (while we used more massive Ar) and a stronger magnetic field than ours. Accordingly, the ion gyroradius can be kept sufficiently small so that the large acceleration by the ICR is possible without the strong ion-wall loss. We expect that, even in our simulation, a larger exhaust velocity can be achieved if the ion wall-loss is kept minimal. We also expect that the PA can optimally contribute 
to the thrust in the absence of the ion wall-loss effect. In the forthcoming papers, we will investigate the thrust by the PA/ICR scheme for wider range of parameters, such as propellant species, device size, magnetic field strength, and number densities of charged and neutral particles. A proof of principle experiment of this scheme has been launched recently at Tokai University.

In order to develop an electrodeless plasma thruster, we should perform self-consistent particle-in-cell (PIC) simulations since the test particle simulations neglect several important ingredients. For example, in the present study, the total penetration of the externally applied rf field into a magnetized plasma is assumed. This is a highly optimistic simplification, and the plasma shielding effect must be taken into account for making more realistic estimates of various quantities. For the development of a Rotating Electric Field (REF) scheme to configure an electrodeless plasma thruster, the problem of the electric field penetration has been investigated in the frequency range between the lower hybrid and electron cyclotron frequencies [6]. For the development of the PA/ICR scheme, we have recently initiated an analysis of the electric field penetration near $\omega=\Omega_{0}$ by using the VORPAL code [19]. The excitation of a spatially localized rf field in magnetized plasmas is also an important experimental problem. The ponderomotive effect on electrons and the self-consistent interaction between rf fields and plasma particles [20] are also important issues for the development of the PA/ICR scheme.

\section{Acknowledgements}

This work has been supported by the Grants-in-Aid for Scientific Research under Contract No. (S) 21226019 from the Japan Society for the Promotion of Science and by Kyushu University Interdisciplinary Programs in Education and Projects in Research Development (P\&P).

\section{Appendix A. Quasi-linear Theory of Cyclotron Heating}

We derive the energy gain due to the ion cyclotron resonance by the quasi-linear theory, reffering to Stix [15]. When the amplitude of the rf electric field is uniform, the perpendicular components of the equation of motion for ions Eq. (2) are given by,

$$
\begin{aligned}
\frac{\mathrm{d} v_{x}}{\mathrm{~d} t}-\Omega(z[t]) v_{y} & =\frac{q}{m_{\mathrm{Ar}}} E_{0} \sin (\omega t), \\
\frac{\mathrm{d} v_{y}}{\mathrm{~d} t}+\Omega(z[t]) v_{x} & =0,
\end{aligned}
$$

where $\Omega(z[t])=q B(z[t]) / m_{\mathrm{Ar}}$ and we simply write $\Omega(z[t])=\Omega(t)$ hereafter. For the convenience of the calculation, we introduce the following complex variable, $u=v_{x}+i v_{y}$; then, Eqs. (A.1a) and (A.1b) become

$$
\begin{aligned}
\frac{\mathrm{d} u}{\mathrm{~d} t}+i \Omega(t) u & =\frac{q E_{0}}{m_{\mathrm{Ar}}} \sin (\omega t) \\
& \simeq \frac{i q E_{0}}{2 m_{\mathrm{Ar}}} \mathrm{e}^{-i \omega t},
\end{aligned}
$$

where we neglect the non-resonant driving term in the last equality. When $E_{0}=0$, the solution of Eq. (A.2) is $u(t)=$ $u\left(t_{0}\right) \exp \left[-i \int_{t_{0}}^{t} \Omega\left(t^{\prime}\right) \mathrm{d} t^{\prime}\right]$. Accordingly, the general solution of Eq. (A.2) for $E_{0} \neq 0$ can be written as

$$
u(t)=u_{0}(t) \exp \left[-i \int_{t_{0}}^{t} \Omega\left(t^{\prime}\right) \mathrm{d} t^{\prime}\right] .
$$

Now we calculate $u_{0}(t)$. Inserting Eq. (A.3) into Eq. (A.2), we obtain

$$
\dot{u}_{0}(t)=\frac{i q E_{0}}{2 m_{\mathrm{Ar}}} \mathrm{e}^{-i \omega t} \exp \left[i \int_{t_{0}}^{t} \Omega\left(t^{\prime}\right) \mathrm{d} t^{\prime}\right] .
$$

If we expand $\Omega(t)$ near the resonance point $z_{0}$ and retain only a linear term, we obtain

$$
\begin{aligned}
& \Omega(t) \simeq \omega+\left.\frac{\mathrm{d} \Omega}{\mathrm{d} t}\right|_{t_{\mathrm{res}}}\left(t-t_{\mathrm{res}}\right), \\
& \left.\Omega^{\prime} \equiv \frac{\mathrm{d} \Omega}{\mathrm{d} t}\right|_{t_{\mathrm{res}}}=\frac{\mathrm{d} \Omega}{\mathrm{d} z} v_{z}=\frac{\omega v_{0 \|}}{L_{\mathrm{B}}},
\end{aligned}
$$

where $t_{\text {res }}=z_{0} / v_{0 \|}$ and $v_{z}$ is assumed to be the initial axial velocity of $v_{0 \|}$. Then, Eq. (A.4) becomes

$$
\dot{u}_{0}(t) \simeq \frac{i q E_{0}}{2 m_{\mathrm{Ar}}} \mathrm{e}^{-i\left\{\omega t_{0}+\Omega^{\prime}\left(t_{0}-t_{\mathrm{res}}\right)^{2} / 2\right\}} \mathrm{e}^{i\left(t-t_{\mathrm{res}}\right)^{2} \Omega^{\prime} / 2} .
$$

If we use the following mathematical formula

$$
\int_{t_{0}}^{t} \mathrm{e}^{i\left(t^{\prime}-t_{\mathrm{res}}\right)^{2} \Omega^{\prime} / 2} \mathrm{~d} t^{\prime}=\left|\frac{2 \pi}{\Omega^{\prime}}\right|^{1 / 2} \mathrm{e}^{i \frac{\pi}{4} \operatorname{sgn}\left(\Omega^{\prime}\right)}
$$

then the solution of $u_{0}(t)$ is written as

$$
\begin{aligned}
& u_{0}(t)=u_{0}\left(t_{0}\right)+\frac{i q E_{0}}{2 m_{\mathrm{Ar}}}\left|\frac{2 \pi}{\Omega^{\prime}}\right|^{1 / 2} e^{-i \psi}, \\
& \text { where } \psi=\omega t_{0}+\frac{\Omega^{\prime}}{2}\left(t_{0}-t_{\mathrm{res}}\right)^{2}-\frac{\pi}{4} \operatorname{sgn}\left(\Omega^{\prime}\right) .
\end{aligned}
$$

Inserting Eq. (A.8a) into Eq. (A.3) and taking an average over the phase, then we finally obtain the perpendicular energy gain of ions as

$$
\begin{aligned}
\Delta \epsilon_{\perp} & =\frac{m_{\mathrm{Ar}}}{2}\left\langle u(t) u^{*}(t)-u\left(t_{0}\right) u^{*}\left(t_{0}\right)\right\rangle \\
& =\frac{\pi q^{2} E_{0}^{2}}{4 m_{\mathrm{Ar}}} \frac{L_{\mathrm{B}}}{\omega v_{0} \|} .
\end{aligned}
$$

[1] K. Toki, S. Shinohara, T. Tanikawa, T. Hada, I. Funaki, K.P. Shamrai, Y. Tanaka and A. Yamaguchi, J. Plasma Fusion Res. SERIES 8, 25 (2009).

[2] S. Shinohara, T. Hada, T. Motomura, K. Tanaka, T. Tanikawa, K. Toki, Y. Tanaka and K.P. Shamrai, Phys. Plasmas 16, 057104 (2009).

[3] H. Nishida, S. Shinohara, T. Tanikawa, T. Hada, I. Funaki, I. Matsuoka, K.P. Shamrai and T. Motomura, 46th AIAA/ASME/SAE/ASEE Joint Propul. Conf. \& Exhibit, AIAA2010-7013, Nashville (2010). 
[4] H. Nishida, S. Shinohara, T. Tanikawa, T. Hada, I. Funaki, T. Matsuoka, F. Otsuka, K.P. Shamrai, T. Motomura, T. Nakamura and T.S. Rudenko, proceedings of Plasma 2011, Kanazawa, Japan (2011).

[5] S. Shinohara, H. Nishida, K. Yokoi, T. Nakamura, T. Tanikawa, T. Hada, F. Otsuka, T. Motomura, E. Ohno, I. Funaki, T. Matsuoka, K.P. Shamrai and T.S. Rudenko, 32nd Int. Electric Propul. Conf., IEPC-2011-056, Wiesbaden, 11-15 Sept. (2011).

[6] T. Matsuoka, I. Funaki, T. Nakamura, K. Yokoi, H. Nishida, T.S. Rudenko, K.P. Shamrai, T. Tanikawa, T. Hada and S. Shinohara, Plasma Fusion Res. 6, 2406103 (2011).

[7] E.A. Bering III, F.R. Chang Díaz, J.P. Squire, T.W. Glover, M.D. Carter, G.E. McCaskill, B.W. Longmier, M.S. Brukardt, W.J. Chancery and V.T. Jacobson, Phys. Plasmas 17, 043509 (2010).

[8] C. Charles, Plasma Sources Sci. Technol. 16, R1 (2007).

[9] I.Y. Dodin, N.J. Fisch and J.M. Rax, Phys. Plasmas 11, 5046 (2004).

[10] A. Ando, K. Hattori and M. Inutake, Proceedings of Space Plasma Symposium 2005, pp.64-69, in Japanese
(2006).

[11] A. Ando, Y. Hosokawa, M. Hatanaka, H. Tobari, K. Hattori and M. Inutake, Thin Solid Films 506-507, 601 (2006).

[12] G. Emsellem, 29th Int. Electric Propul. Conf., IEPC-2005156, Princeton (2005).

[13] B.M. Lamb, G. Dimonte and G.J. Morales, Phys. Fluids 27, 1401 (1984).

[14] Y. Arakawa, H. Kuninaka, Y. Nakayama and K. Nishiyama, Ion engines for powered flight in space (Corona Publishing Co., LTD., 2006) (in Japanese).

[15] T.H. Stix, Waves in plasmas (Springer-Verlag New York, Inc., 1992) p.485.

[16] V. Vahedi and M. Surendra, Comput. Phys. Commun. 87, 179 (1995).

[17] W.H. Cramer, J. Chem. Phys. 30, 641 (1959).

[18] M.L. Vestal, C.R. Blakley and J.H. Futrell, Phys. Rev. A 17, 17 (1978).

[19] http://www.txcorp.com/products/VORPAL/

[20] B.N. Breizman and A.V. Arefiev, Phys. Plasmas 8, 907 (2001). 